The Astronomical Journal, 122:3136-3154, 2001 December

(C) 2001. The American Astronomical Society. All rights reserved. Printed in U.S.A.

\title{
NEAR-INFRARED PHOTOMETRY OF GLOBULAR CLUSTERS NGC 6287 AND NGC 6341 (M92): THE FORMATION OF THE GALACTIC HALO ${ }^{1}$
}

\author{
JAE-Woo LeE ${ }^{2}$ AND BruCE W. CARneY \\ Department of Physics and Astronomy, University of North Carolina, Chapel Hill, NC 27599-3255; jaewoo@astro.unc.edu, bruce@physics.unc.edu \\ LAURA K. FUlltoN \\ Observatoire de Genève, CH-1290 Sauverny, Switzerland; Laura.Fullton@obs.unige.ch
}

AND

Peter B. Stetson

Dominion Astrophysical Observatory, Herzberg Institute of Astrophysics, National Research Council, 5071 West Saanich Road, Victoria, BC V8X 4M6, Canada; peter.stetson@hia.nrc.ca Received 2001 August 1; accepted 2001 September 20

\begin{abstract}
We present Hubble Space Telescope (HST) Near Infrared Camera and Multi-Object Spectrometer (NICMOS) camera 3 photometry of the inner halo globular cluster NGC 6287 and the intermediate halo globular cluster NGC 6341 (M92). Our photometric measurements range from the lower red giant branch to $\approx 3$ mag below the main-sequence turnoff, allowing us to define accurate mean loci of the two clusters. Our relative age estimate between NGC 6287 and M92 using the color difference between the main-sequence turnoff and the base of the red giant branch shows that they essentially have the same ages within \pm 2 Gyr. The slightly different chemical compositions between the two clusters do not significantly influence our result. Our simulations using synthetic spectra show that the temperature dependence of the interstellar reddening law for the HST NICMOS photometric system could be a more serious problem in the relative age estimate using the color difference between the main-sequence turnoff and the base of the red giant branch. However, this effect adds no more than $\pm 0.5-1$ Gyr to the uncertainty in our relative age estimate. Our color-magnitude diagram of NGC 6287 shows a well-defined blue horizontal branch population. This is consistent with its age and metallicity. We also present the relative interstellar reddening toward NGC 6287 and distance modulus with respect to those of M92. We find $E(B-V)=0.62$ and $(m-M)_{0}=14.35$ for NGC 6287, which are generally in good agreement with previous estimates. Our photometry suggests that there exists a differential reddening across NGC 6287 with an amount of $\Delta E(B-V)=0.07-0.09 \mathrm{mag}$, consistent with the previous study of Stetson \& West. The Galactocentric distance of NGC 6287 becomes $R_{\mathrm{GC}}=1.6 \mathrm{kpc}$ if $R_{0}=8.0 \mathrm{kpc}$. Based on our new radial velocity measurement for NGC 6287, the cluster's (minimum) apogalacticon distance is $R_{a} \approx 5.5$ kpc, suggesting that NGC 6287 is an inner halo cluster. Within this framework, our results are consistent with the idea that the globular cluster formation must have been triggered everywhere at the same time in our Galaxy. In the appendices, we discuss that the interstellar extinction law of the HST NICMOS photometric system, particularly in the F110W passband, depends on the temperature of the stars in the sense that hotter stars suffer more interstellar absorption than cooler stars.
\end{abstract}

Key words: dust, extinction - Galaxy: formation - Galaxy: halo -

Galaxy: kinematics and dynamics - globular clusters: individual (NGC 6287, NGC 6341)

\section{INTRODUCTION}

When did our Galaxy form? In what regions did star formation first begin? How rapidly did the star formation proceed in regions of different densities? Understanding how the oldest stellar populations formed in our Galaxy has always been one of the key quests in modern astrophysics. It would be wonderful if one could approach this problem observationally by studying the star formation histories of the distant galaxies or more distant quasars with large sample sizes. Because of the observational limitations,

\footnotetext{
${ }^{1}$ Based on observations made with the NASA/ESA Hubble Space Telescope, obtained at the Space Telescope Science Institute, which is operated by the Association of Universities for Research in Astronomy, Inc., under NASA contract NAS 5-26555.

${ }^{2}$ Current address: Center for Space Astrophysics, Yonsei University, Shinchon-dong 134, Seoul 120-749, Korea, and PMA Division, Mail Stop 405-47, California Institute of Technology, Pasadena, CA 91125; jaewoo@srl.caltech.edu.
}

unfortunately, the first generations of star formation are not observable even for the highest-redshift quasars (Hamann $\&$ Ferland 1999). Therefore we eventually have to dig down into the oldest fossils in our own Galaxy, in particular by studying the ages, dynamics, and chemical compositions of the globular cluster systems.

Galaxy formation theories may be categorized by two classical models and their variants: the rapid coherent Galaxy formation model of Eggen, Lynden-Bell, \& Sandage (1962, hereafter ELS) and the slow chaotic Galaxy formation model of Searle \& Zinn (1978, hereafter SZ). An age distribution of globular cluster systems with a narrow peak and a long, sparsely populated tail toward younger ages would favor an ELS-style Galaxy formation model (e.g., Stetson, VandenBerg, \& Bolte 1996; Richer et al. 1996; Rosenberg et al. 1999), while an age spread of several gigayears in the globular cluster systems would be more consistent with the framework pictured by SZ (e.g., Lee, Demarque, \& Zinn 1994; Chaboyer, Demarque, \& Sarajedini 1996; Sarajedini, Chaboyer, \& Demarque 1997). 
The real history of our Galaxy formation may reside somewhere between the two. For example, van den Bergh (1996) proposed that the central region of the Galaxy system collapsed from a single protogalaxy à la ELS, while the outer part of the Galaxy is thought to have been assembled by infall and subsequent capture of protogalactic fragments, as envisioned by SZ.

During the last decade, a tremendous amount of information has been accumulated regarding the ages of globular cluster systems. The Hubble Space Telescope (HST) observations of one of the most remote halo clusters NGC 2419 $\left(R_{\mathrm{GC}}=90 \mathrm{kpc}\right)$ of Harris et al. (1997) have shown that NGC 2419 and $\mathrm{M} 92\left(R_{\mathrm{GC}}=9.6 \mathrm{kpc}\right)$ essentially have the same age within \pm 2 Gyr, suggesting that globular cluster formation must have started everywhere at about the same time in our Galaxy (Richer et al. 1996; see also the counterargument of Chaboyer et al. 1996). Moreover, the local dwarf galaxies Carina (Mighell 1997), Draco (Grillmair et al. 1998), Leo I and II (Held et al. 2000; Mighell \& Rich 1996), and Ursa Minor (Mighell \& Burke 1999) and the globular clusters in the Fornax dwarf galaxy (Buonanno et al. 1998), LMC (Johnson et al. 1999), and the Sagittarius dwarf galaxy (Montegriffo et al. 1998; Layden \& Sarajedini 2000) appear to be coeval with typical globular clusters of comparable metallicity in our Galaxy. These comparable ages suggest that globular cluster formation may have begun everywhere at the same time not only in our Galaxy but also in the local dwarf galaxies, despite the differences in the initial physical environments.

The "inside-out" galaxy formation scenario suggests that the star formation must have started from the high-density regions of the galaxy because of the smaller free collapse timescale $\tau \propto \rho^{-1 / 2}$ near the central part of our Galaxy. This idea led van den Bergh (1993) to suggest that NGC 6287 may be the oldest globular cluster in our Galaxy, based on its metallicity, HB population, and its spatial location in our Galaxy.

Stetson \& West (1994) were the first to present $B V$ CCD photometry of NGC 6287, and they confirmed that it has a blue horizontal branch (BHB). However, they questioned whether the cluster is an inner halo cluster. Hesser, Shawl, \& Meyer (1986) measured the radial velocity of NGC 6287, $v_{r}=-208 \mathrm{~km} \mathrm{~s}^{-1}$, by using the low-resolution image tube spectra. Stetson \& West (1994) noted that NGC 6287 may have sufficient kinetic energy to take it out as far as the solar circle. Since their color-magnitude diagram (CMD) for NGC 6287 did not reach below the main-sequence turnoff (TO), they could not measure the age of the cluster.

Davidge (1998) and Davidge \& Courteau (1999) presented $J H K$ photometry of NGC 6287. The photometry of Davidge \& Courteau (1999) reached $\approx 1$ mag below the TO and they suggested that the age difference between NGC 6287 and M92 is no larger than \pm 2 Gyr.

Finally, Fullton, Stetson, \& Carney (1999) presented HST Wide Field Planetary Camera 2 (WFPC2) photometry of NGC 6287. Their preliminary results indicated that NGC 6287 and NGC 2419 (Harris et al. 1997) have the same age but slightly different RGB and HB morphologies.

In this paper, we explore the age difference between NGC 6287 and M92 by using HST Near Infrared Camera and Multi-Object Spectrometer (NICMOS) camera 3 (NIC3) photometry. As is well known, M92 is one of the oldest globular clusters in our Galaxy and it lies at an intermediate Galactocentric distance $\left(R_{\mathrm{GC}}=9.6 \mathrm{kpc}\right)$. Since these two clusters appear to have comparable metallicities $(\delta[\mathrm{Fe} /$ $\mathrm{H}] \approx 0.2 \mathrm{dex}$ in the sense of M92 being more metal-poor), an accurate relative age estimate can be achieved using the method proposed by VandenBerg, Bolte, \& Stetson (1990).

\section{OBSERVATIONS AND DATA REDUCTIONS}

The observations for NGC 6287 and M92 were carried out on 1998 January 29 (UT) and 1998 January 12 (UT), respectively, using HST NIC3 during the "first NIC3 campaign." NIC3 is a $256 \times 256$ pixel $\mathrm{HgCdTe}$ array. The field of view (FOV) is $51^{\prime \prime} .2 \times 51^{\prime \prime} 2$, which gives a scale of 0 ".2 pixel $^{-1}$ (f/17.2). All the images were obtained through the F110W and F160W filters using the multiple accumulate (MULTIACCUM) mode (Calzetti et al. 1999). For each cluster, images at two different locations were obtained. For NGC 6287, the locations of the central positions of the two fields are $35^{\prime \prime}$ south (field F1; $\alpha=17^{\mathrm{h}} 5^{\mathrm{m}} 9^{\mathrm{s}}, \delta=-22^{\circ} 43^{\prime} 4^{\prime \prime}$ ) and $46^{\prime \prime}$ north (field F2; $\alpha=17^{\mathrm{h}} 5^{\mathrm{m}} 12^{\mathrm{s}}, \delta=-22^{\circ} 41^{\prime} 59^{\prime \prime}$ ) of the cluster center. For M92, the fields are 31" south (field F3; $\alpha=17^{\mathrm{h}} 17^{\mathrm{m}} 7^{\mathrm{s}}, \delta=43^{\circ} 7^{\prime} 41^{\prime \prime}$ ) and $106^{\prime \prime}$ south (field F4; $\left.\alpha=17^{\mathrm{h}} 17^{\mathrm{m}} 5^{\mathrm{s}}, \delta=43^{\circ} 6^{\prime} 28^{\prime \prime}\right)$ of the cluster center. Since the offset values from each cluster center are much smaller than the tidal radii of the two clusters (see Table 1) and the FOV is rather small, our object frames should well reflect the clusters' stellar populations. For each field, the spiral pattern dithering (SPIRAL-DITH), which produces a spiral around the first pointing, at three positions with a 4.5 pixel $(0,9)$ offset, was applied to improve flat-fielding, avoid bad pixels, and reduce the effects of the undersampled pointspread function (PSF). In Table 2, we present the journal of observations. In the table, we list the field identification, the passband, the date and time (UT), the total integration time, the sample sequence name, and the number of the sample sequence in the MULTIACCUM mode ${ }^{3}$ for each image.

All images have been processed using the standard NICMOS calibration pipeline procedure (CALNICA in STSDAS/IRAF ${ }^{4}$ ) to perform bias subtraction, bad-pixel identification, dark-current subtraction, linearity correction, flat-field correction, photometric calibration, cosmicray identification and correction, and identification of saturated pixels (Calzetti et al. 1999). Generally, these

\footnotetext{
${ }^{3}$ Because of the nondestructive nature of the NICMOS readout, NICMOS enables us to read out intermediate pixel values at specific times during an integration. The intermediate readout times are defined in Calzetti et al. (1999), depending on the sample sequences.

${ }^{4}$ IRAF (Image Reduction and Analysis Facility) is distributed by the National Optical Astronomy Observatory, which is operated by the Association of Universities for Research in Astronomy, Inc., under contract with the National Science Foundation.
}

TABLE 1

Cluster Properties

\begin{tabular}{ccccccc}
\hline \hline ID & {$[\mathrm{Fe} / \mathrm{H}]$} & $E(B-V)$ & $l$ & $b$ & $\begin{array}{c}R_{\mathrm{GC}} \\
(\mathrm{kpc})\end{array}$ & $\begin{array}{c}r_{t}^{\mathrm{a}} \\
(\operatorname{arcsec})\end{array}$ \\
\hline NGC $6287 \ldots$ & $-2.00^{\mathrm{b}}$ & 0.62 & 0.13 & 11.02 & 1.6 & 631 \\
$\mathrm{M} 92 \ldots \ldots \ldots$ & $-2.25^{\mathrm{c}}$ & 0.02 & 68.34 & 34.86 & 9.5 & 912 \\
\hline
\end{tabular}

${ }^{\text {a }}$ The tidal radius (Trager, King, \& Djorgovski 1995).

b Based on the high-resolution echelle spectroscopy of the two giants in NGC 6287 (Lee \& Carney 2001).

${ }^{\mathrm{c}}$ Sneden et al. 1991. 
TABLE 2

JOURNAL OF OBSERVATIONS

\begin{tabular}{|c|c|c|c|c|c|c|}
\hline ID, Field & Filter & Date & $\begin{array}{c}\text { Time } \\
\text { (UT Start) }\end{array}$ & $\begin{array}{l}t_{\text {exp }} \\
(\mathrm{s})\end{array}$ & Sequence Name & No. Samp. \\
\hline \multicolumn{7}{|l|}{ NGC 6287: } \\
\hline F1 ....... & F110W & 1998 Jan 29 & 0410 & 255.95 & STEP256 & 11 \\
\hline $\mathrm{F} 1 \ldots \ldots$ & F160W & 1998 Jan 29 & 0415 & 319.95 & STEP64 & 13 \\
\hline $\mathrm{F} 1 \ldots \ldots$ & F110W & 1998 Jan 29 & 0422 & 223.96 & STEP32 & 14 \\
\hline $\mathrm{F} 1 \ldots \ldots$ & F160W & 1998 Jan 29 & 0426 & 319.95 & STEP64 & 13 \\
\hline $\mathrm{F} 1 \ldots \ldots$ & F110W & 1998 Jan 29 & 0432 & 223.96 & STEP32 & 14 \\
\hline $\mathrm{F} 1 \ldots \ldots$ & F160W & 1998 Jan 29 & 0436 & 319.95 & STEP64 & 13 \\
\hline $\mathrm{F} 2 \ldots \ldots$ & F110W & 1998 Jan 29 & 0442 & 111.93 & STEP16 & 13 \\
\hline $\mathrm{F} 2 \ldots \ldots$ & F160W & 1998 Jan 29 & 0444 & 143.92 & STEP16 & 15 \\
\hline $\mathrm{F} 2 \ldots \ldots$ & F110W & 1998 Jan 29 & 0447 & 111.93 & STEP16 & 13 \\
\hline $\mathrm{F} 2 \ldots \ldots$ & F160W & 1998 Jan 29 & 0449 & 143.92 & STEP16 & 15 \\
\hline $\mathrm{F} 2 \ldots \ldots$ & F110W & 1998 Jan 29 & 0453 & 111.93 & STEP16 & 13 \\
\hline $\mathrm{F} 2 \ldots \ldots$ & F160W & 1998 Jan 29 & 0455 & 143.92 & STEP16 & 15 \\
\hline \multicolumn{7}{|l|}{ M92: } \\
\hline F3 ...... & F110W & 1998 Jan 12 & 2144 & 159.96 & STEP32 & 12 \\
\hline F3..... & F160W & 1998 Jan 12 & 2147 & 255.96 & STEP256 & 11 \\
\hline F3 ...... & F110W & 1998 Jan 12 & 2152 & 159.96 & STEP32 & 12 \\
\hline F3 ...... & F160W & 1998 Jan 12 & 2155 & 255.96 & STEP256 & 11 \\
\hline F3..... & F110W & 1998 Jan 12 & 2200 & 159.96 & STEP32 & 12 \\
\hline F3..... & F160W & 1998 Jan 12 & 2203 & 255.96 & STEP256 & 11 \\
\hline $\mathrm{F} 4 \ldots \ldots$ & F110W & 1998 Jan 12 & 2209 & 159.96 & STEP32 & 12 \\
\hline $\mathrm{F} 4 \ldots \ldots$ & F160W & 1998 Jan 12 & 2212 & 255.95 & STEP256 & 11 \\
\hline $\mathrm{F} 4 \ldots \ldots$ & F110W & 1998 Jan 12 & 2217 & 159.96 & STEP32 & 12 \\
\hline $\mathrm{F} 4 \ldots \ldots$ & F160W & 1998 Jan 12 & 2220 & 255.95 & STEP256 & 11 \\
\hline $\mathrm{F} 4 \ldots \ldots$ & F110W & 1998 Jan 12 & 2225 & 127.96 & STEP64 & 10 \\
\hline $\mathrm{F} 4 \ldots \ldots$ & F160W & 1998 Jan 12 & 2228 & 191.96 & STEP32 & 13 \\
\hline
\end{tabular}

procedures produced almost perfectly flattened object frames. However, the spider diffraction spikes around the bright stars in NIC3 showed asymmetries as previously reported by Krist et al. (1998) in NIC2. In all frames, the spikes from upper left to lower right show stronger features than those from top right to bottom left in the NICMOS coordinate system. In Figure 1, we present the science images for NGC 6287 and M92.

To fully use the dynamic range provided by the MULTIACCUM mode in NICMOS, we used science images and data quality flag arrays for our analyses at the short $(\approx 2 \mathrm{~s})$, the intermediate $(\approx 16 \mathrm{~s})$, and the longest integration time $\left(t_{\exp }\right.$ in Table 1$)$ from each exposure sequence.

PSF photometry for all frames was performed with DAOPHOTII/ALLSTAR and ALLFRAME (Stetson 1994, 1995; Turner 1995), following the procedures described in Lee \& Carney (1999). The first step was to run the FIND routine on each object frame, with the detection threshold set at $5 \sigma$ above the local sky background level. During the FIND routine, the fraction of the accidental inclusion of faint elliptical galaxies ${ }^{5}$ in our star lists is expected to be high, especially near M92 because of its higher galactic latitude $\left(b=35^{\circ}\right)$. It should be noted that, because of the undersampled nature of NIC3 data, it is almost impossible to distinguish faint elliptical galaxies from stars in our object frames using photometric indices, such as a roundness or a sharpness returned from DAOPHOT's FIND routine. It is likely that the only feasible way to distinguish

\footnotetext{
${ }^{5}$ The recent deep H-band galaxy counts by Yan et al. (1998) suggest that approximately six galaxies are included in our object frames at $m_{160}=21 \mathrm{mag}$ and 16 galaxies at $23 \mathrm{mag}$.
}

faint elliptical galaxies from stars is by using their colors. After the FIND routine, we examined by eye the positions of star lists superposed on each object frame and removed probable nonstellar objects detected on the diffraction spikes of the bright stars, which extend to several arcseconds on the image. The aperture photometry was performed with star lists thus generated. PSFs were then calculated from isolated bright stars. To select good PSF stars, we rejected stars containing saturated pixels, hot or cold pixels, or bad pixels determined during the calibration pipeline procedure, by making use of the data quality flag arrays. At least five iterations were required to remove neighboring stars from our PSF stars to achieve good PSFs on each object frame. For the PSF computations, we adopted a spatially invariable PSF. The existence of asymmetric features in the diffraction spikes may indicate that there exists an unknown problem in the NICMOS optics (Krist et al. 1998), but it is not known whether it varies with the position on the detector or not. Since the NIC3 object frames were undersampled, the effect of the PSF undersampling in the stellar profile appears to overwhelm the variation in PSFs with their positions (whether it exists or not) on each object frame. Also the number of PSF stars is too small to use variable PSFs on our object frames. Therefore, a nonvariable PSF should be adequate for our analyses. After the first ALLSTAR pass, we examined the subtracted images and added undetected stars to our star lists, and they were used as input star lists for the second ALLSTAR run.

To construct the CMDs, we used ALLFRAME. We derived positional transformation solutions for each dithered-exposure F110W and F160W pair by using DAOMATCH and DAOMASTER, and then we combined them together with MONTAGE2. Using the combined 


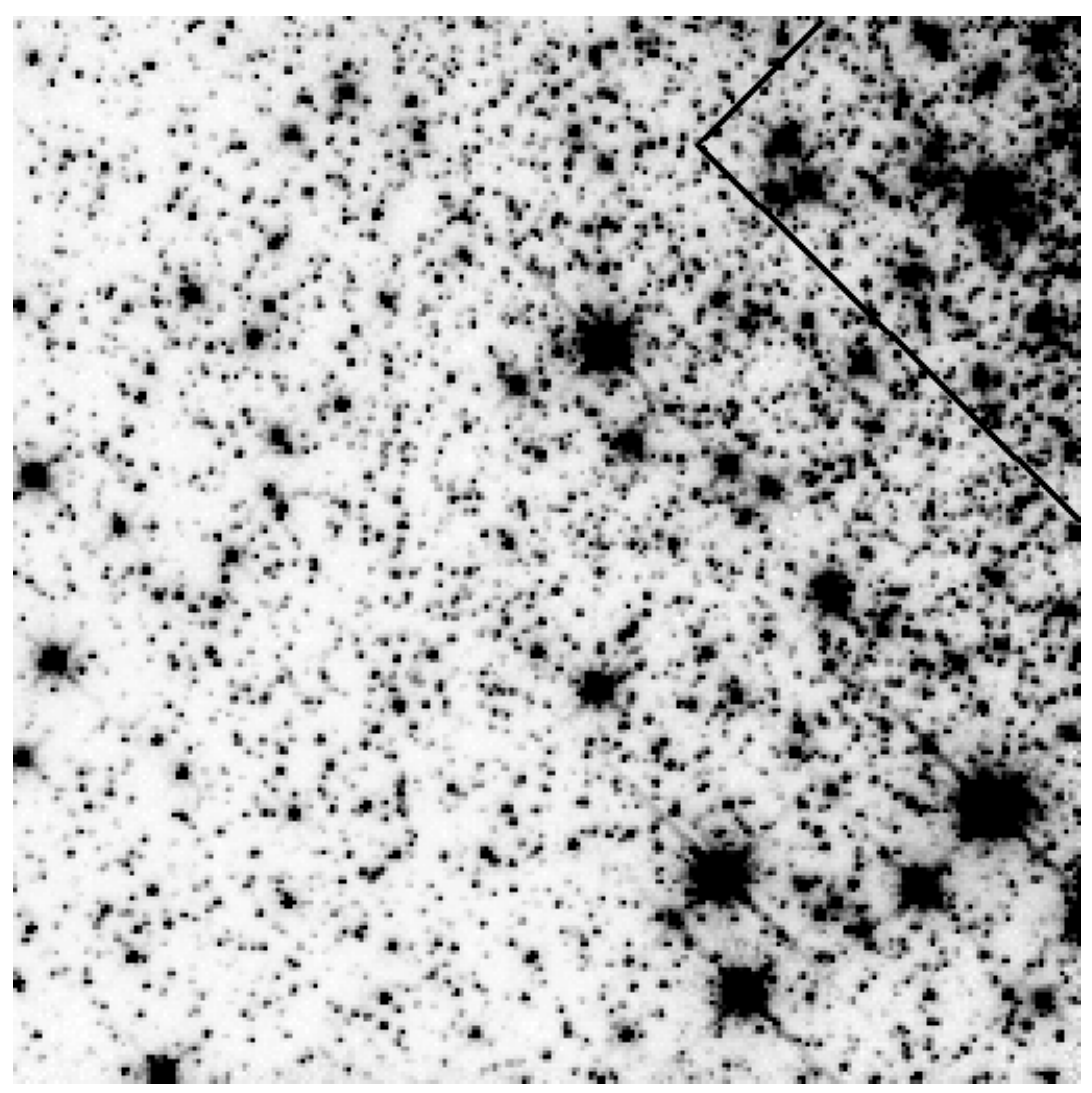

FIG. $1 a$

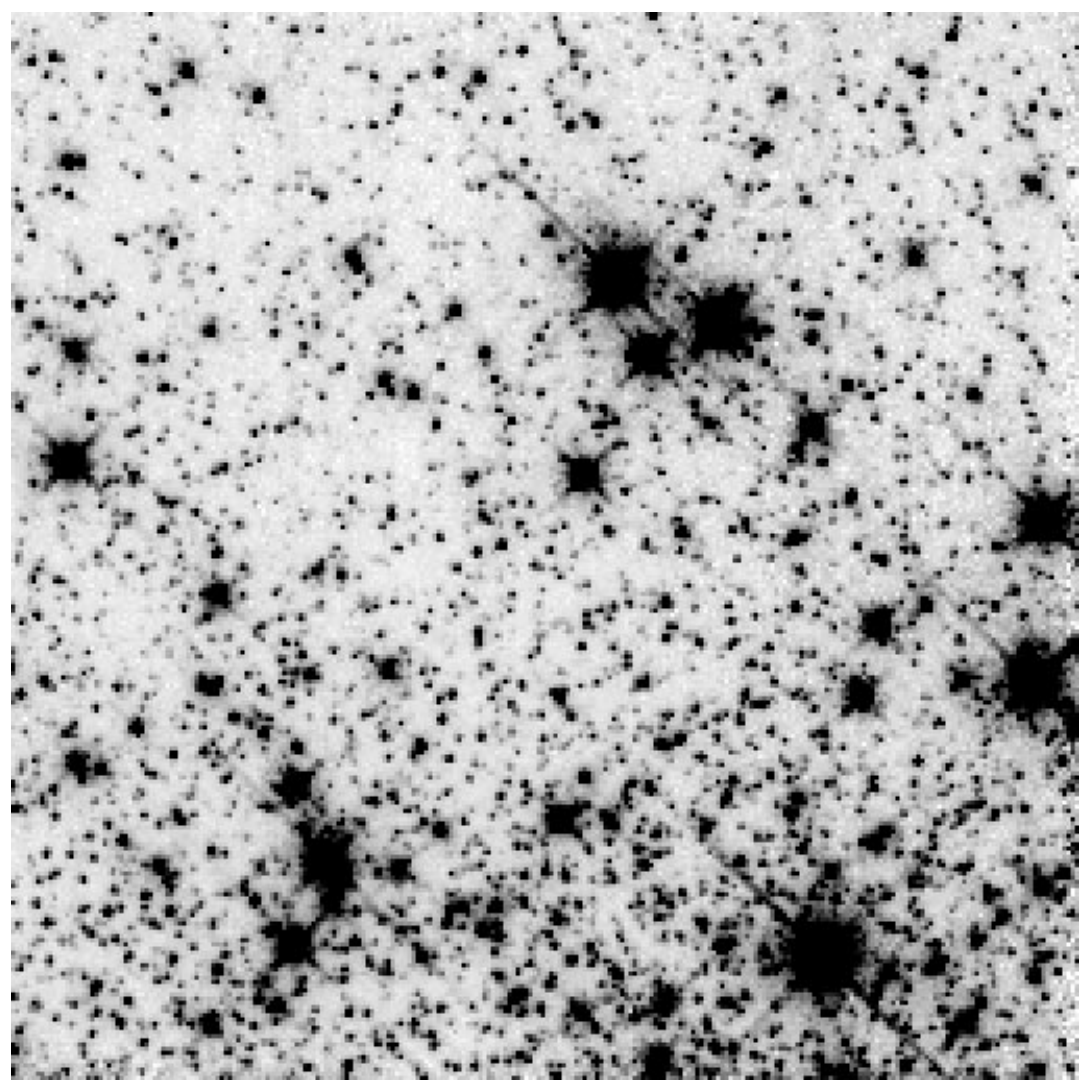

FIG. $1 b$

Fig. 1.-Final F160W images of NGC 6287 fields $(a)$ F1 and $(b)$ F2, and M92 fields $(c)$ F3 and $(d)$ F4. For NGC 6287, northeast is at the top, and northwest is to the right. The overlapped area with Davidge \& Courteau (1999) is noted with a solid line in the field F1. For M92, north is to the right and east is at the top. 


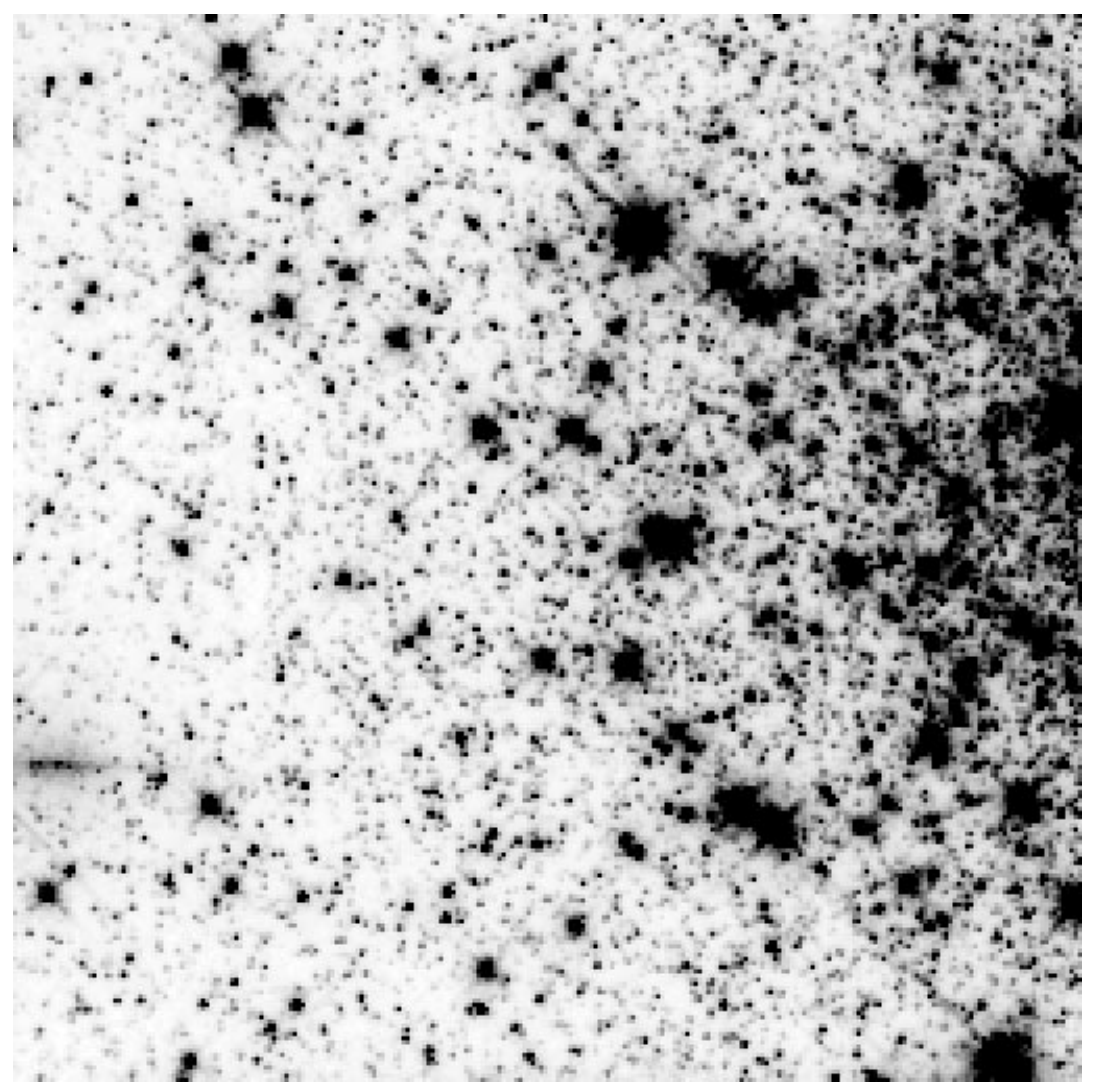

FIG. 1c

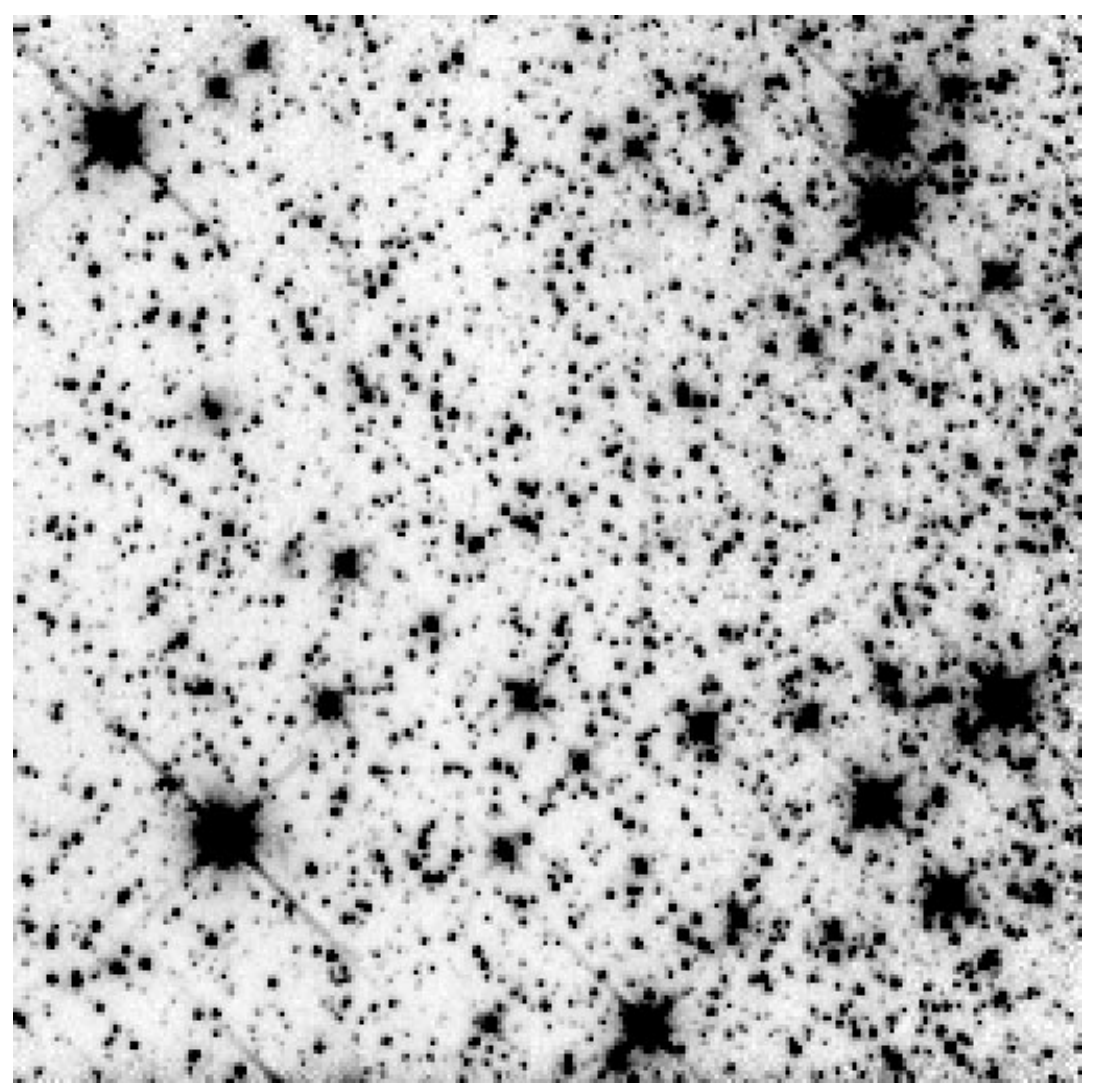

FIG. $1 d$ 
positional information and magnitudes returned from the second ALLSTAR pass as input star lists, ALLFRAME was run on the original images. After the first ALLFRAME run, we examined the subtracted frames by eye to add undetected stars or to reject probable nonstellar objects detected again on the diffraction spikes. At this stage, however, the modification of the input star lists was almost unnecessary. With the final star lists, ALLFRAME was rerun. After the final ALLFRAME run, we performed the aperture correction to correct the total count rates to a $1^{\prime \prime}$ radius aperture, and then these corrected count rates were multiplied by 1.075 to convert to those with a nominal infinite aperture (Calzetti et al. 1999). Then we averaged count rates of the three dithered images from each field to calculate mean count rates of stars. During these calculations, we compared the data quality flag arrays to reject stars containing saturated pixels, hot or cold pixels, or bad pixels determined during the calibration pipeline procedure within our fitting radius (1.6 pixels) in the ALLFRAME run. This rejection step reduces the total numbers of useful stars in each object frame. However, we are more interested in accurate magnitudes and colors of stars in both clusters than in deriving accurate number densities, such as a luminosity function.
Through these steps, the total numbers of stars measured were 2120 and 1754 in NGC 6287 fields F1 and F2, respectively. In M92 fields F3 and F4, we measured 1947 and 1074 stars, respectively. Finally, the mean count rates were converted onto the HST magnitude system (STMAG) using the relation

$$
m_{\mathrm{ST}}=-2.5 \log (\text { PHOTFLAM } \times \mathrm{CR})-21.1,
$$

where PHOTFLAM is the inverse sensitivity of the instrumental setup and CR is the count rate measured in unit time.

Although we converted our measurements onto the STMAG system, our results cannot be directly compared with those from NIC1 or NIC2. The major concern in the absolute calibration of NIC3 photometry is the variation in the intrapixel sensitivity combined with the undersampled PSFs, as pointed out by others (see Colina \& Rieke 1997; Lauer 1999; Storrs et al. 1999). It is known that the detector sensitivity varies across individual pixels. The detector sensitivity at the edge of individual pixels can be significantly reduced and this variation in the intrapixel sensitivity is stronger in NICMOS than any other detectors on board $H S T$. The total flux of a stellar image, therefore, can vary
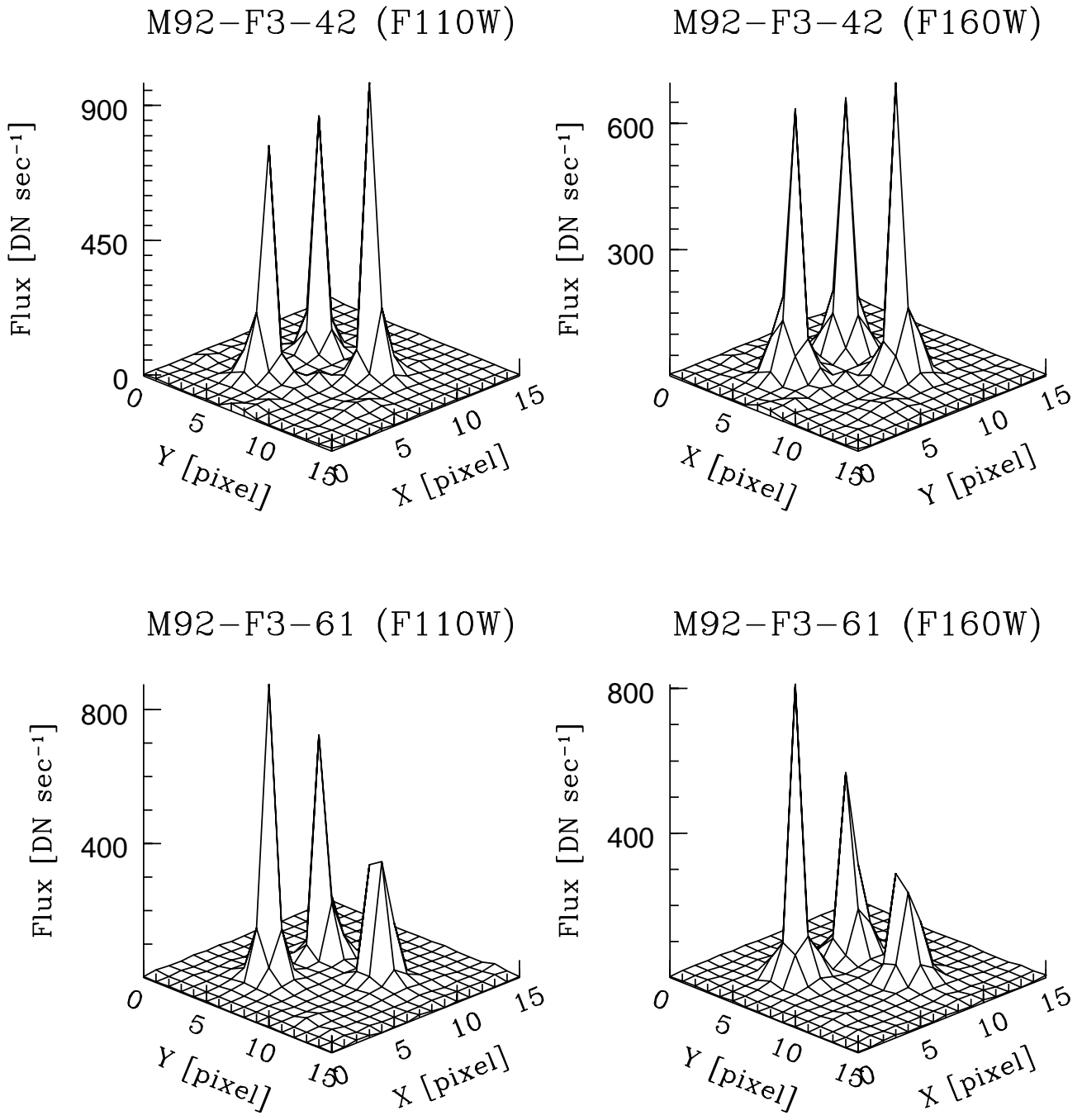

FIG. 2.-Comparisons of normalized fluxes of the two isolated bright stars (M92-F3-42 and M92-F3-61) in M92. In each plot, the left peak is for exposure sequence 1 (Exp-1), the middle for exposure sequence 2 (Exp-2), and the right for exposure sequence 3 (Exp-3). Exp-2 was dithered by 4.5 pixels along the $y$-axis, and Exp-3 was dithered by 4.5 pixels in both axes relative to Exp-1. 

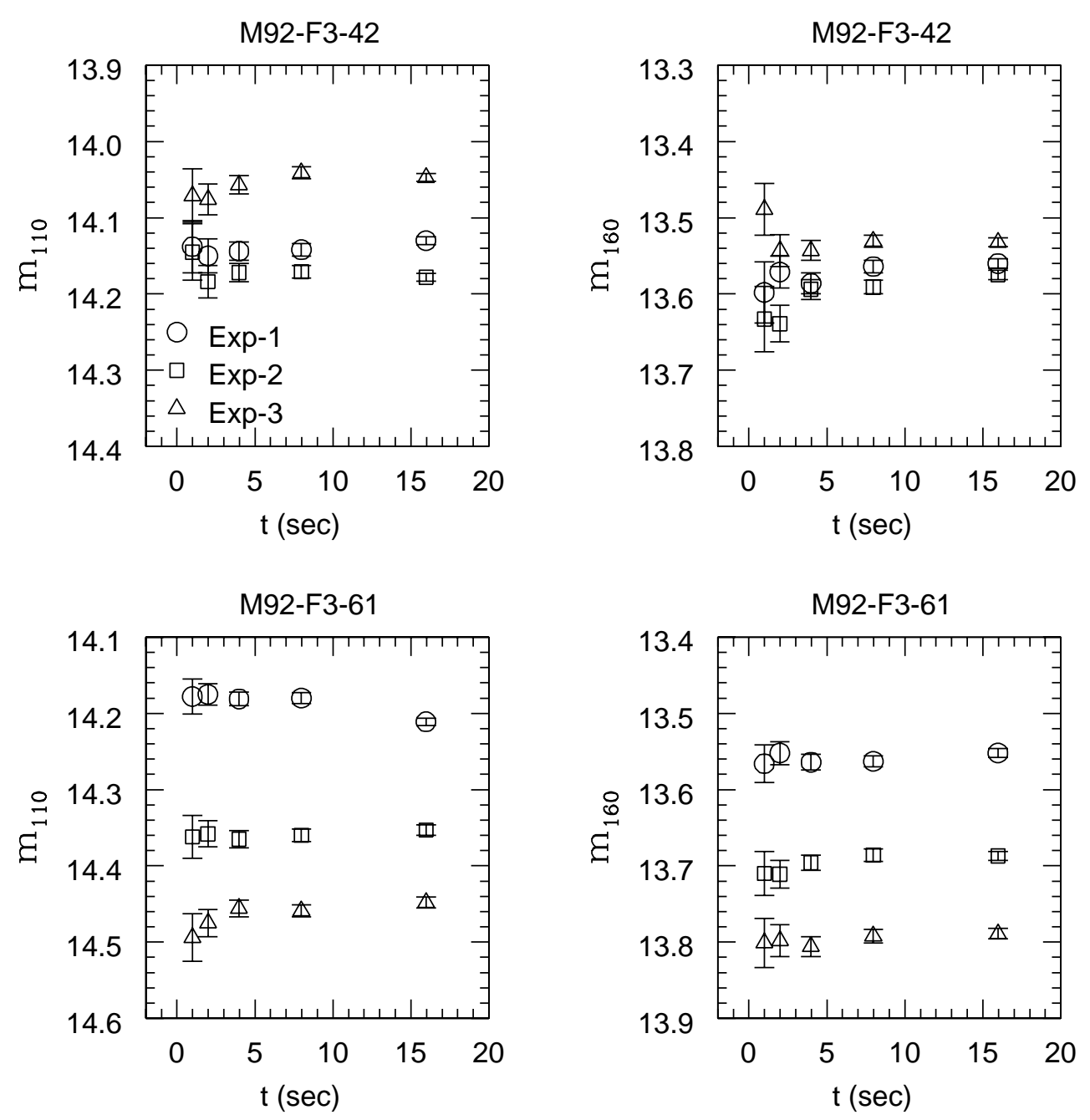

FIG. 3. - Plots of the uncorrected magnitude vs. exposure time. Note that the magnitude difference in the star M92-F3-61 is as large as 0.3 mag.

according to the size and the location of the imaged PSF on individual pixels. This effect is more significant in NIC3 than NIC1 or NIC2 because the size of imaged PSFs in NIC3 is smaller than that of individual pixels (i.e., undersampled). Also this is more significant in the F110W passband than in the F160W passband in NIC3 because of the more centrally concentrated PSF in the F110W. As a result, the total flux of a stellar image may vary by up to $\approx 0.4 \mathrm{mag}$ in NIC3 (Lauer 1999). We examine this effect on an object frame. In Figure 2, we plot two isolated bright stars in M92 (using our star IDs, M92-F3-42 and M92-F361). We combined surface profiles from the three dithered frames together to examine the variation in the stellar flux. As can be seen, the peak flux can vary remarkably from one exposure to another. It should be noted that the variation in the peak flux as shown in the figure can be explained by undersampling alone. For example, when an imaged PSF is located at the edge of the pixel its total flux will be contained over 2 or 4 pixels, depending on its location, while when it is located at the center of the pixel most of the stellar flux will be contained in a single pixel. However, the variation in the measured flux according to the positions on the detector suggests that there is variation in the intrapixel sensitivity. In Figure 3, we plot the variation in the aperture magnitude with 1 " aperture radius by using the same stars as above. We also show the variation of magnitude with integration time in each exposure sequence. Not surprisingly, the magnitudes do not vary with the integration time in one exposure sequence and they agree within the measurement errors. The magnitudes are not constant in the separate pointings. The differences in magnitude range up to $\approx 0.3$ mag in our example. Further examination with an expanded sample also confirms that the variation in the intrapixel sensitivity is the dominant source of the photometric errors in NIC3.

Storrs et al. (1999) have devised an ad hoc correction method for the NIC3 photometry. They introduced a sharpness ratio, which is defined by the ratio of the flux in the peak pixel to the flux in the whole PSF, and found relations between the sharpness ratios and the total flux correction terms for both F110W and F160W passbands. In Figure 4, we plot the variations in the magnitudes after applying the photometric corrections recommended by Storrs et al. (1999). The discrepancies in magnitude are generally reduced; however, they still can vary by up to $\approx 0.1$ mag even in isolated bright stars. Applying this photometric correction method to a crowded field, such as in globular clusters, may be difficult. In practice, since we have to take account of the flux from neighboring stars, measuring sharpness ratios in a crowded field is not an easy task.

It is thought that the effects from the undersampling of the imaged PSF and the variation in the intrapixel sensi- 
M92-F3-42

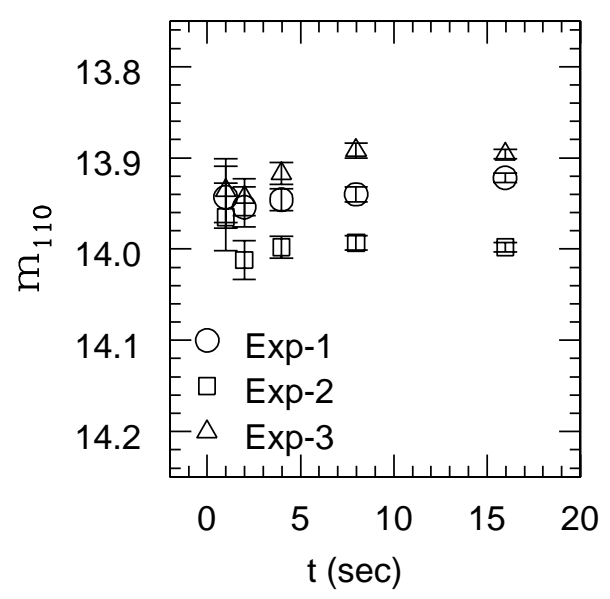

M92-F3-61

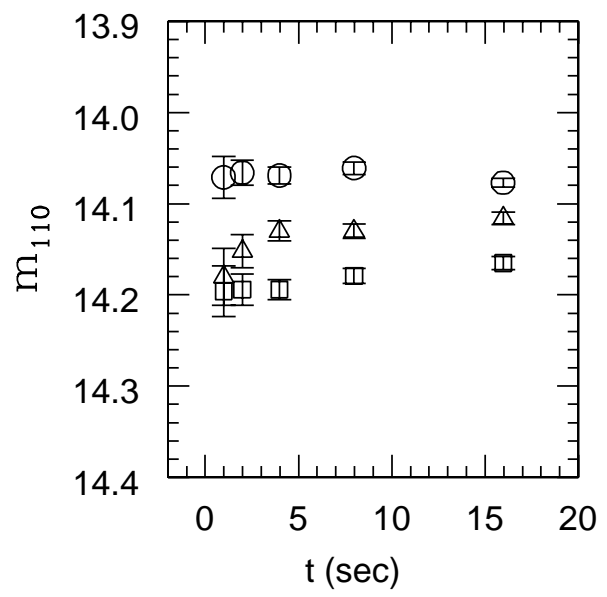

M92-F3-42
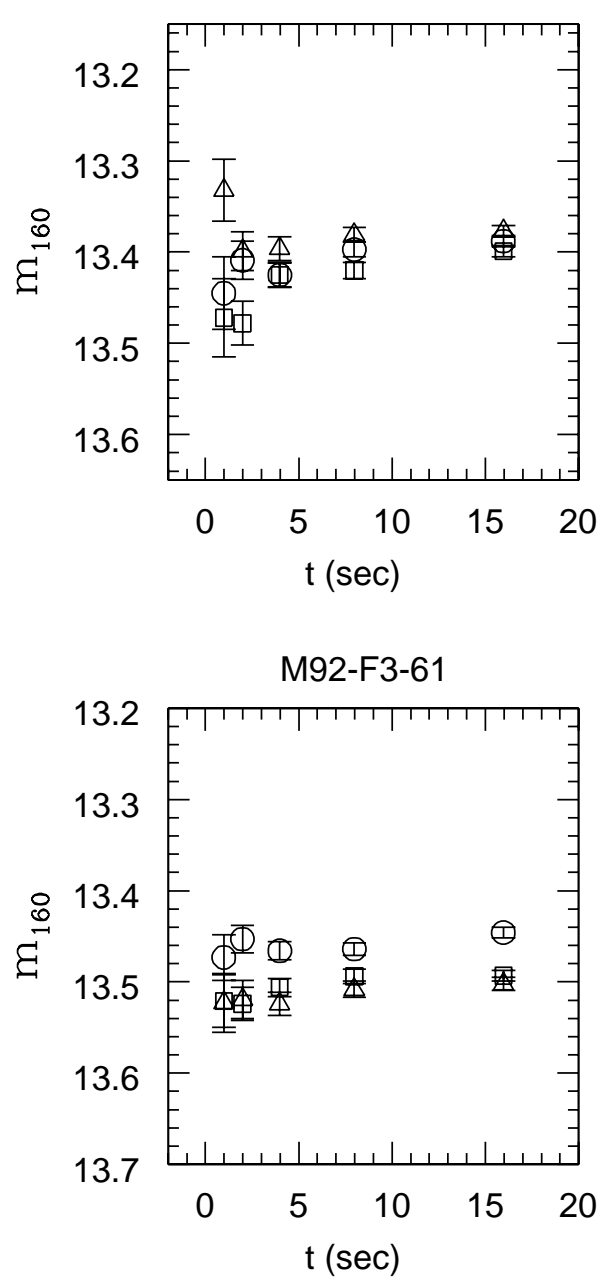

FIG. 4. - Plots of the corrected magnitude vs. exposure time. In the plots, the correction method by Storrs et al. (1999) was applied, and it generally improves the discrepancy of measurements.

tivity do not depend on the magnitude, or total flux, of the stars. In fact, they depend on the size of imaged PSFs and the subpixel position of imaged PSFs on individual pixels. In the case of the relative flux comparisons between globular clusters, for example, these problems can be mitigated. When the number of stars is large enough, the photometric error induced by the variation in the intrapixel sensitivity should have random behavior within a finite size of the distribution, $\approx 0.4 \mathrm{mag}$ at most in NIC3, giving similar mean values and variances in the photometric error distribution functions between clusters, and this effect will be cancelled out when one compares the relative mean magnitudes or colors between clusters. If this is the case, a comparison of mean cluster magnitudes or colors between clusters is still useful in NIC3 photometry.

To test this idea, we performed some artificial-star simulations. We generated well-sampled model NIC3 PSFs for the F110W and F160W passbands by using the latest version of Tiny Tim (Krist \& Hook 2000). Then we randomly distributed 500 stars with a single exponential luminosity function and an adopted fiducial sequence from the main sequence to the giant branch on a blank frame. We prepared two copies of this artificial image for both passbands. We performed PSF photometry on these images, and we fully recovered the input fiducial sequence. In
Figure 5, we present the fiducial sequence with a solid line. (Note that this line does not contain information on the input stellar number density.) Using the first set, we shrank the artificial image until its plate scale matches that of NIC3 $\left(0,2\right.$ pixel $\left.^{-1}\right)$ to simulate undersampling only. For the other set, we convolved the intrapixel sensitivity kernel for a NIC3 isolated pixel (Lauer 1999) and then shrank this convolved image until its pixel size matches with that of NIC3 to simulate both the variation in the intrapixel sensitivity and undersampling. The individual simulated images were set to have the same array size as NICMOS, $256 \times 256$ pixels. For both image sets, we simulated a SPIRAL-DITH type dithering at three positions with a 4.5 pixel offset to maintain an environment similar to that of the real images, but the background noise was not simulated. Then PSF photometry was performed on these artificial frames with DAOPHOTII/ALLSTAR and ALLFRAME, following the same procedures described above. After the ALLFRAME pass, we averaged the magnitudes from the three dithered images to calculate the mean magnitude for both cases.

Our results are shown in Figure 5. The dots represent the result for the undersampled PSF only, and plus signs those for the undersampled PSF convolved with the variation in the intrapixel sensitivity in NIC3. We also show the estimated signal-to-noise ratio $(\mathrm{S} / \mathrm{N})$. Our simulations suggest 


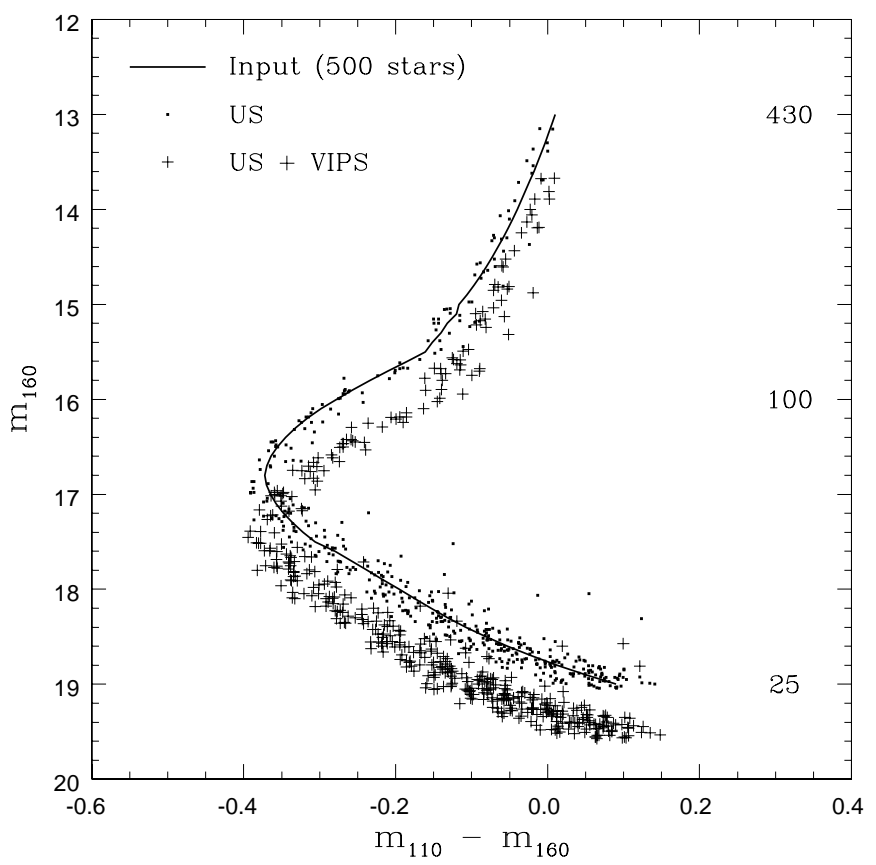

FIG. 5.-Surrogated fiducial sequence with Salpeter mass function $(\alpha=2.35)$ in our simulation (solid line), the measurement of the undersampling simulated images (US; dots), and the measurement of the undersampling and the variation of the intrapixel sensitivity kernel simulated images (US + VIPS; plus signs). The numbers on the right-hand side represent the estimated $\mathrm{S} / \mathrm{N}$ ratio of the input magnitude.

that undersampling is mainly responsible for broadening the magnitudes and colors in the output sequence, while the variation in the intrapixel sensitivity is responsible for shifting magnitudes and colors in the output sequence, i.e., lowering the observed flux in both passbands. The scatter in color around the mean fiducial sequence for the images convolved with the variation in the intrapixel sensitivity and undersampling appear to be slightly larger than those
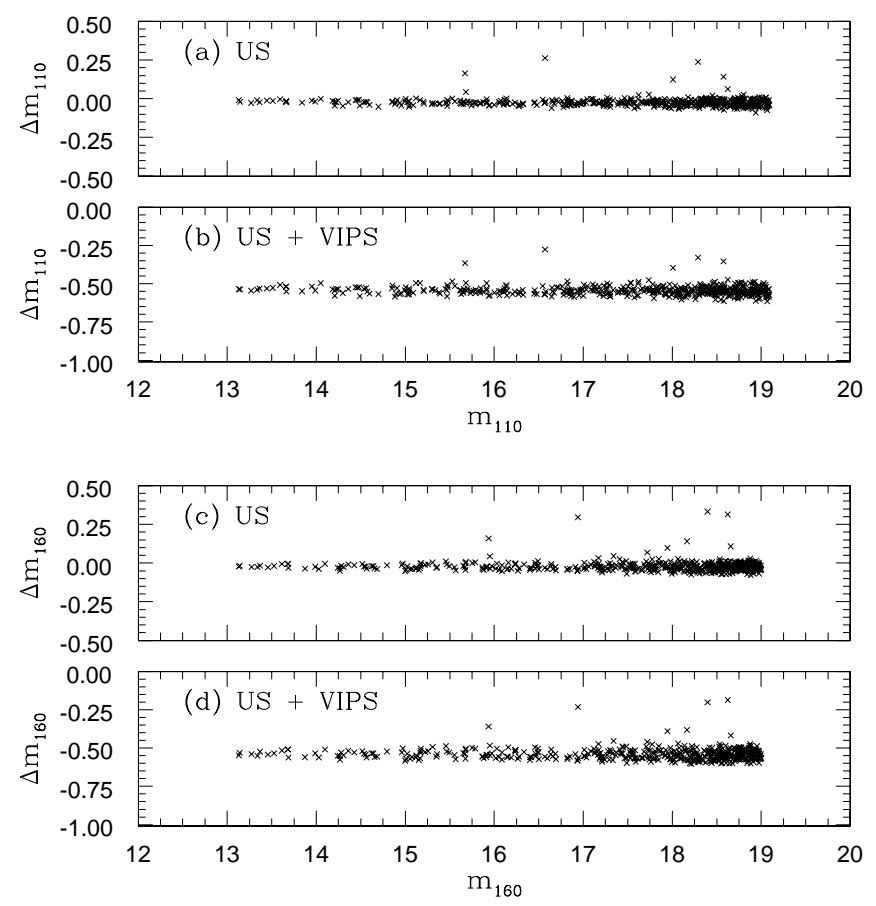

FIG. 6.- Residuals as a function of input magnitude. The differences are in the sense of input magnitude minus output magnitude.

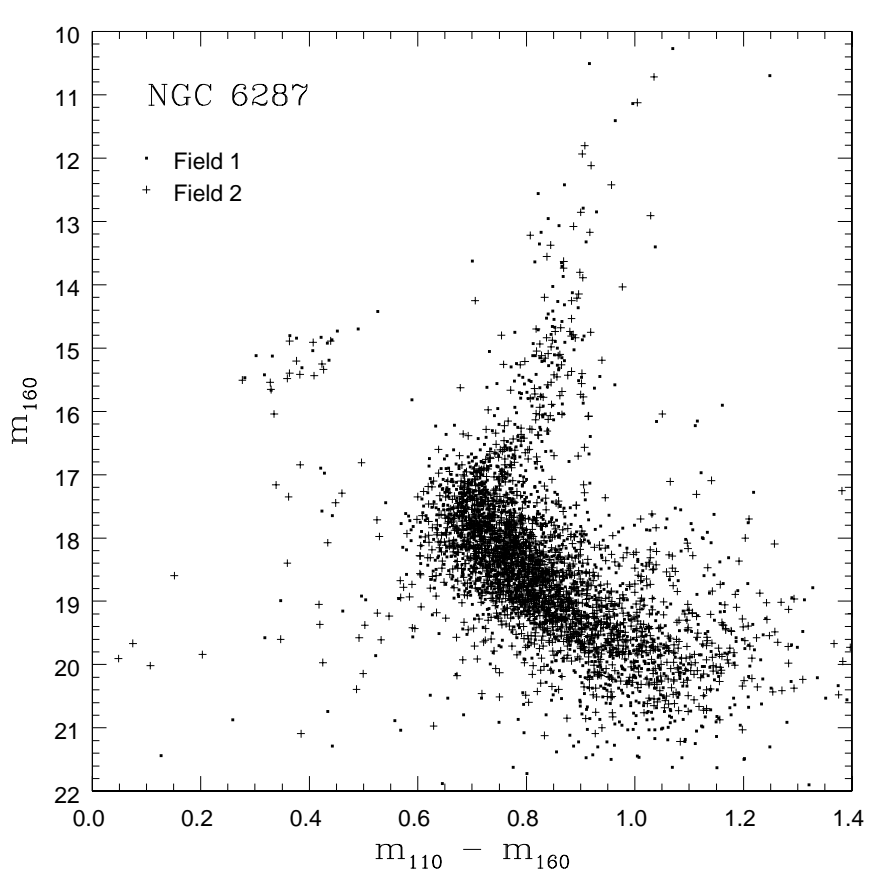

FIG. 7.-Composite color-magnitude diagram for NGC 6287, showing the stars in field F1 (dots) and the stars in field F2 (plus signs). The differential reddening in field $\mathrm{F} 2$ was corrected with $\Delta E\left(m_{110}-m_{160}\right)=0.04 \mathrm{mag}$ and $\Delta A_{m_{160}}=0.05 \mathrm{mag}$.

for undersampling only, but the differences are negligibly small. The residuals, in the sense of input magnitudes minus output magnitudes, are shown as a function of input magnitudes in Figure 6. As can be seen, the residuals are almost flat around the mean values, and there are no gradients in residuals in both passbands, indicating that main-sequence fitting between globular clusters is still useful in NIC3 photometry; nevertheless, the absolute calibration of the NIC3 photometry remains uncertain. We performed more simulations by adding more stars in the artificial frames.

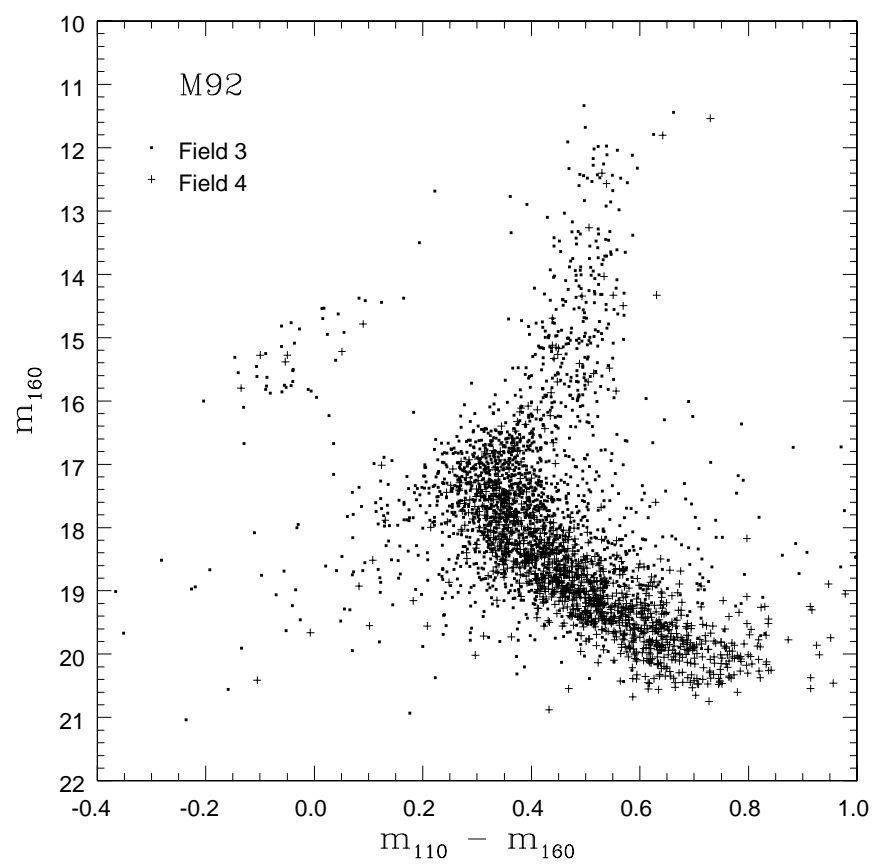

FIG. 8.-Composite color-magnitude diagram for M92, showing stars in field F3 (dots) and in field F4 (plus signs). 


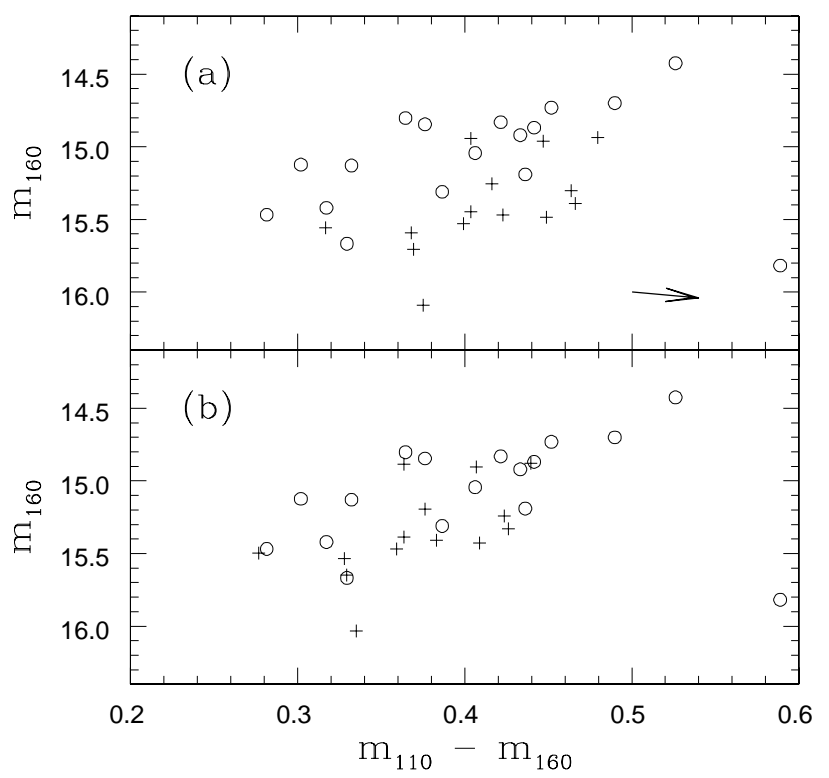

FIG. 9.-Color-magnitude diagrams of the HB region in NGC 6287 (a) without the differential reddening correction, and $(b)$ with the differential reddening correction. HB stars in field F1 are represented by circles and those in field $\mathrm{F} 2$ by plus signs. The differential reddening vector in field $\mathrm{F} 2$ is indicated by an arrow in $(a)$. The location of HB stars is defined better with the differential reddening correction.

The measurement error due to the crowding becomes evident as increasing the number of stars, but the results remain the same.

\section{RESULTS}

\subsection{Color-Magnitude Diagrams}

We present CMDs for NGC 6287 and M92 by using the STMAG system in Figures 7 and 8, respectively. It should be noted that the mean colors between fields F1 and F2 in NGC 6287 do not agree each other. We compared the mean loci of the main sequence for the two fields, which were obtained by using a combination of subjective removal of outliers followed by use of iterative sigma-clipping mean color calculations. We obtained the mean color difference $\Delta\left(m_{110}-m_{160}\right)=0.04 \pm 0.01 \mathrm{mag}$ in the sense that the mean color in F2 is redder than in F1. (The error is the $1 \sigma$ level.) In case of M92, the mean colors between the fields F3 and F4 show good agreement. This suggests that the difference in the mean colors of the NGC 6287 fields is due to differential reddening.

Visual inspection of the Palomar Observatory Sky Survey prints showed that the distribution of interstellar clouds around NGC 6287 is rather patchy, suggesting the existence of the differential reddening. This was also pointed out by Stetson \& West (1994). They presented the positions of BHB stars around the cluster center with $B-V$ colors in their Figure 7. The mean $B-V$ colors of BHB stars toward the field $\mathrm{F} 2\left(\approx 46^{\prime \prime}\right.$ north from the cluster center) appear to be redder than those of $F 1\left(\approx 35^{\prime \prime}\right.$ south from the cluster center), which is consistent with our result. They also derived a mean reddening gradient $\delta(B-V)=+0.0016$ mag $\operatorname{arcsec}^{-1}$ in the sense that the interstellar reddening increases with declination. Using their relation, the amount of the differential reddening between fields $F 1$ and $F 2$ could be as large as $\Delta E(B-V)=0.13 \mathrm{mag}$, which is equivalent to $\Delta E(J-H)=0.04 \mathrm{mag}$, by adopting the interstellar extinction law by Rieke \& Lebofsky (1985; see below).

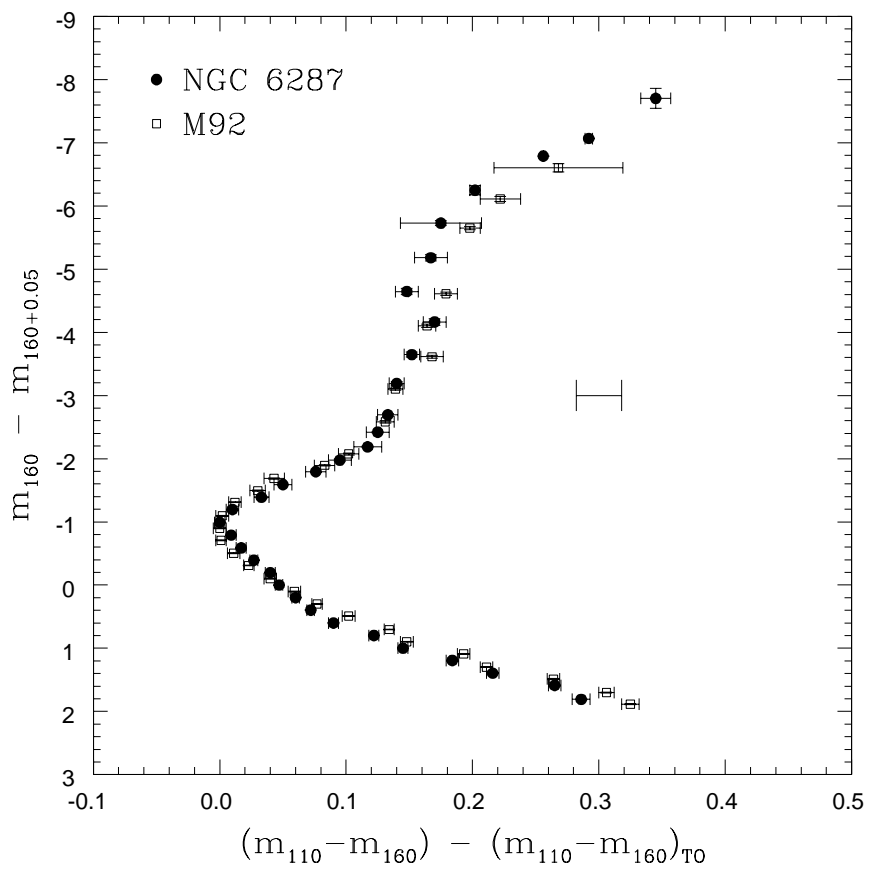

FIG. 10.-Plot of $\left(m_{110}-m_{160}\right)-\left(m_{110}-m_{160}\right)_{\text {To }}$ vs. $m_{160}-m_{160+0.05}$ for fiducial sequences of NGC 6287 and M92, showing the fiducial sequence of NGC 6287 (circles) and the M92 fiducial sequence (squares). The isolated error bar on the right-hand side indicates the difference in color of the giant branch with $\pm 2 \mathrm{Gyr}$ of the age difference.

Therefore, we conclude that the color difference $\Delta\left(m_{110}\right.$ $\left.-m_{160}\right)=0.04$ mag probably results from the differential reddening between the two fields. We therefore have forced the mean color of stars in the field F2 to match that in the field F1. The magnitude-correction term in the F160W passband $\Delta m_{160}$ was also estimated. By using the relation given by Origlia \& Leitherer (2000) the color difference $\Delta\left(m_{110}-m_{160}\right)=0.04 \mathrm{mag}$ is equivalent to $\Delta(J-H) \approx 0.03$ mag in the color range of our interest. Employing the interstellar extinction law, $E(J-H)=0.33 E(B-V)$ and $A_{H}=$ $0.54 E(B-V)$ (Rieke \& Lebofsky 1985), we obtained $\Delta E(B-V)=0.09 \pm 0.03 \mathrm{mag}$ and $\Delta A_{H}=0.05 \pm 0.02 \mathrm{mag}$ $\left(\approx \Delta A_{m_{160}}\right)$. Although our differential reddening estimation $\Delta E(B-V)=0.09 \mathrm{mag}$ is slightly smaller than that of Stetson \& West (1994), the results agree within the uncertainties.

In Appendix A, we present the interstellar extinction estimates for the F110W and F160W passbands. Using these relations for the main sequence TO in Table $6, E\left(m_{110}\right.$ $\left.-m_{160}\right)=0.59 E(B-V)$, the color difference between the fields $\mathrm{F} 1$ and $\mathrm{F} 2$ is equivalent to $\Delta E(B-V)=0.07 \mathrm{mag}$, which is slightly smaller than that from using the extinction laws for $J H$ passbands.

In Figure 9, we show CMDs of the HB region of NGC 6287 before and after applying the differential reddening correction. We also note the differential reddening vector between the two fields in the figure. As can be seen in Figure $9 b$, applying the differential reddening correction improves the agreement in the distribution of HB stars between the two fields; in particular it better defines the location of the lower envelope of HB stars in the CMD.

\subsection{Main-Sequence Fitting and Relative Age Estimation}

Our fiducial sequences from the main sequence to the giant branch for NGC 6287 and M92 are given in Tables 3 
TABLE 3

FiduCIAL SEQUENCE FOR NGC 6287

\begin{tabular}{|c|c|c|}
\hline$m_{160}$ & $m_{110}-m_{160}$ & $n$ \\
\hline $10.496 \pm 0.158$ & $1.053 \pm 0.017$ & 2 \\
\hline $11.131 \pm 0.005$ & $1.000 \pm 0.003$ & \\
\hline $11.410 \pm 0.000$ & $0.964 \pm 0.000$ & . \\
\hline $11.951 \pm 0.075 .$. & $0.910 \pm 0.004$ & \\
\hline $12.471 \pm 0.038$. & $0.883 \pm 0.032$ & \\
\hline $13.019 \pm 0.050$. & $0.875 \pm 0.013$ & \\
\hline $13.554 \pm 0.051$ & $0.856 \pm 0.009$ & \\
\hline $14.035 \pm 0.053 .$. & $0.878 \pm 0.009$ & \\
\hline $14.552 \pm 0.039 .$. & $0.860 \pm 0.006$ & 18 \\
\hline $15.007 \pm 0.026 .$. & $0.848 \pm 0.006$ & 37 \\
\hline $15.506 \pm 0.024$ & $0.841 \pm 0.008$ & 39 \\
\hline $15.779 \pm 0.013$ & $0.833 \pm 0.009$ & 18 \\
\hline $16.013 \pm 0.014$ & $0.825 \pm 0.011$ & 20 \\
\hline $16.222 \pm 0.017$ & $0.803 \pm 0.009$ & 14 \\
\hline $16.404 \pm 0.013$ & $0.784 \pm 0.008$ & 21 \\
\hline $16.609 \pm 0.009 \ldots \ldots$ & $0.758 \pm$ & 32 \\
\hline $16.810 \pm 0$ & $0.741=$ & 52 \\
\hline $17.003 \pm 0$ & $0.718=$ & 69 \\
\hline $17.208 \pm 0$ & $0.708 \pm$ & 108 \\
\hline $17.409 \pm 0.005 \ldots \ldots$ & $0.717 \pm$ & 131 \\
\hline $17.612 \pm 0.005 \ldots \ldots$ & $0.725 \pm 0.004$ & 149 \\
\hline $17.806 \pm 0.004 \ldots \ldots$ & $0.735 \pm 0.003$ & 188 \\
\hline $18.002 \pm 0.004 \ldots$ & $0.748 \pm 0.004$ & 180 \\
\hline $18.199 \pm 0.004 \ldots$ & $0.755 \pm 0.003$ & 215 \\
\hline $18.398 \pm 0.004 \ldots$ & $0.768 \pm 0.003$ & 208 \\
\hline $18.596 \pm 0.004 \ldots$ & $0.780 \pm 0.003$ & 219 \\
\hline $18.799 \pm 0.004 \ldots \ldots$ & $0.798 \pm 0.004$ & 192 \\
\hline $18.997 \pm 0.004 \ldots$. & $0.830 \pm 0.004$ & 19 \\
\hline $19.199 \pm 0.005 \ldots$ & $0.853 \pm 0.004$ & 154 \\
\hline $19.392 \pm 0.005 \ldots$ & $0.892 \pm 0.005$ & 125 \\
\hline $19.594 \pm 0.005 \ldots$ & $0.924 \pm 0.005$ & 115 \\
\hline $19.787 \pm 0.005 \ldots \ldots$ & $0.973 \pm 0.005$ & 97 \\
\hline $20.008 \pm 0.007 \ldots \ldots$ & $0.994 \pm 0.007$ & 62 \\
\hline
\end{tabular}

and 4, respectively. Note that column (5) in the tables represents the number of stars used in our calculations, not the clusters' luminosity functions.

We explore the difference in age using, in essence, color differences between the base of the RGB (BRGB) and the TO. VandenBerg et al. (1990) described a method to derive accurate relative cluster ages with similar chemical compositions. They recommended shifting the clusters' CMDs in color until the TO colors agreed, then shifting the magnitudes until the upper main sequence is matched at a point 0.05 mag redder than the TO. The color differences of the resulting RGBs could then be used to estimate the relative ages, since the color difference between the TO and the $\mathrm{BRGB}$ is a monotonic and inverse function of age. Further, this color difference is independent of distance, reddening, ${ }^{6}$ and photometric zero-point errors. This method appears to be nearly independent of metallicity within moderately large ranges in $[\mathrm{Fe} / \mathrm{H}]$, although there is some sensitivity to differences in elemental abundance ratios, such as $[\alpha / \mathrm{Fe}]$. This method can also be employed using infrared photometry. The infrared color difference between the BRGB and the TO, for instance $\left(m_{110}-m_{160}\right)_{\mathrm{BRGB}}-\left(m_{110}-m_{160}\right)_{\mathrm{TO}}$, is a monotonic and inverse function of age at a given metallicity, since the difference in the effective temperature

${ }^{6}$ In fact, this method does depend on the reddening for the HST NICMOS photometric system. See below.
TABLE 4

FidUCIAL SEQUENCE FOR M92

\begin{tabular}{|c|c|c|}
\hline$m_{160}$ & $m_{110}-m_{160}$ & $n$ \\
\hline $11.499 \pm 0.064 \ldots \ldots$ & $0.597 \pm 0.051$ & 4 \\
\hline $11.993 \pm 0.039 \ldots \ldots$ & $0.551 \pm 0.016$ & 10 \\
\hline $12.452 \pm 0.027 \ldots \ldots$ & $0.527 \pm 0.008$ & 17 \\
\hline $13.493 \pm 0.028 \ldots \ldots$ & $0.508 \pm 0.009$ & 27 \\
\hline $14.003 \pm 0.027 \ldots \ldots$ & $0.493 \pm 0.007$ & 29 \\
\hline $14.491 \pm 0.025 \ldots \ldots$ & $0.497 \pm 0.009$ & 38 \\
\hline $15.003 \pm 0.017 \ldots \ldots$ & $0.468 \pm 0.006$ & 55 \\
\hline $15.523 \pm 0.019 \ldots$ & $0.460 \pm 0.007$ & 56 \\
\hline $16.029 \pm 0.017 .$. & $0.431 \pm 0.008$ & 74 \\
\hline $16.209 \pm 0.010 \ldots$ & $0.412 \pm 0.008$ & 27 \\
\hline $16.418 \pm 0.009 \ldots \ldots$ & $0.372 \pm 0.008$ & 41 \\
\hline $16.610 \pm 0.007 \ldots \ldots$ & $0.359 \pm 0.006$ & 60 \\
\hline $16.794 \pm 0.006 \ldots \ldots$ & $0.341 \pm 0.005$ & 77 \\
\hline $17.006 \pm 0.006 \ldots \ldots$ & $0.331 \pm 0.005$ & 100 \\
\hline $17.208 \pm 0.006 \ldots \ldots$ & $0.329 \pm 0.005$ & 93 \\
\hline $17.398 \pm 0.005 \ldots \ldots$ & $0.330 \pm 0.004$ & 127 \\
\hline $17.601 \pm 0.005 \ldots \ldots$ & $0.340 \pm 0.005$ & 121 \\
\hline $17.794 \pm 0.005 \ldots \ldots$ & $0.352 \pm 0.004$ & 126 \\
\hline $18.003 \pm 0.006 \ldots \ldots$ & $0.369 \pm 0.005$ & 117 \\
\hline $18.205 \pm 0.005 \ldots \ldots$ & $0.388 \pm 0.005$ & 122 \\
\hline $18.403 \pm 0.005 \ldots \ldots$ & $0.406 \pm 0.004$ & 135 \\
\hline $18.594 \pm 0.005 \ldots \ldots$ & $0.431 \pm 0.005$ & 115 \\
\hline $18.808 \pm 0.005 \ldots \ldots$ & $0.463 \pm 0.004$ & 122 \\
\hline $19.000 \pm 0.005 \ldots$. & $0.477 \pm 0.005$ & 106 \\
\hline $19.193 \pm 0.006 \ldots \ldots$ & $0.522 \pm 0.005$ & 101 \\
\hline $19.401 \pm 0.005 \ldots \ldots$ & $0.540 \pm 0.005$ & 106 \\
\hline $19.591 \pm 0.007 \ldots \ldots$ & $0.593 \pm 0.005$ & 79 \\
\hline $19.807 \pm 0.007 \ldots \ldots$ & $0.635 \pm 0.006$ & 73 \\
\hline $19.989 \pm 0.008 \ldots \ldots$ & $0.654 \pm 0.007$ & 54 \\
\hline
\end{tabular}

between the BRGB and the TO, in fact, is a monotonic and inverse function of age (see, for example, the theoretical isochrones in the infrared passbands of Bell 1992). Similar to the visual passband, at a point $\Delta\left(m_{110}-m_{160}\right)=0.05$ mag redder than the main-sequence TO, $m_{160+0.05}$, the clusters' main-sequence (MS) fiducial sequences have a considerable slope and little curvature, and therefore it is easier to determine $m_{160+0.05}$ magnitude than that of TO.

In Figure 10, we present a diagram of $\left(m_{110}-m_{160}\right)$ $-\left(m_{110}-m_{160}\right)_{\text {To }}$ versus $m_{160}-m_{160+0.05}$ for NGC 6287 and M92 by using the fiducial sequences in Tables 3 and 4 . In the figure, the two fiducial sequences show excellent agreement from 3 mag below the TO to $1 \mathrm{mag}$ above the BRGB. We used the oxygen-enhanced theoretical model isochrones for $[\mathrm{Fe} / \mathrm{H}]=-2.26$ by Bergbusch \& VandenBergh (1992) for calibrating the relative ages. Using the color-temperature relations given by Bessell, Castelli, \& Plez (1998), the $\left(\log T_{\text {eff }}, \log L\right)$-plane was converted into the $(J-H, H)$-plane, and then we obtained the color difference from the BRGB to the TO change rate of $\Delta(J-H) /$ $\Delta \tau \approx 0.01 \mathrm{mag} \mathrm{Gyr}^{-1}$ over the 10 to $16 \mathrm{Gyr}$ age range for $[\mathrm{Fe} / \mathrm{H}]=-2.26$. We derived the inverse relation ${ }^{7}$ between

\footnotetext{
${ }^{7}$ This inverse relation gives about twice as large a value as the inverse relation of Stephens et al.,$$
m_{110}-m_{160}=0.36+1.04(J-H)
$$

(2000, eq. [5]). The relation given by Stephens et al. (2000) is based on the comparison of metal-rich giants in Baade's window, between HST NIC2 and the ground-based observations.
} 
TABLE 5

List of Previous Reddening and Distance Modulus

\begin{tabular}{cccccr}
\hline \hline \multicolumn{2}{c}{ NGC 6287} & & \multicolumn{2}{c}{ M92 } & \\
\cline { 1 - 2 }$E(B-V)$ & $(m-M)_{0}$ & & $E(B-V)$ & $(m-M)_{0}$ & Ref. \\
\hline $0.62 \ldots \ldots$ & 14.00 & & 0.03 & 14.41 & 1 \\
$0.32 \ldots \ldots$ & $\ldots$ & & 0.01 & $\ldots$ & 2 \\
$0.51 \ldots \ldots$ & $\ldots$ & & 0.02 & $\ldots$ & 3 \\
$0.56 \ldots \ldots$ & $\ldots$ & & 0.03 & $\ldots$ & 4 \\
$0.55 \ldots \ldots$ & 14.16 & & 0.02 & 14.38 & 5 \\
$0.65 \ldots \ldots$ & $14.1-14.4$ & $\ldots$ & $\ldots$ & 6 \\
$0.60 \ldots \ldots$ & 14.65 & & 0.02 & 14.58 & 7 \\
& $\ldots$ & & 0.03 & 14.82 & 8 \\
& $\ldots$ & & 0.02 & 14.61 & 9 \\
& $\ldots$ & 0.02 & 14.87 & 10 \\
\hline
\end{tabular}

REFERENCES. - (1) Zinn 1980; (2) Burstein \& Heiles 1982; (3) Webbink 1985; (4) Reed, Hesser, \& Shawl 1988; (5) Djorgovski 1993; (6) Stetson \& West 1994; (7) Harris (June 1999 version) 1996; (8) Gratton et al. 1997; (9) Pont et al. 1998; (10) Reid 1997.

$J-H$ and $m_{110}-m_{160}$ of Origlia \& Leitherer (2000),

$$
\begin{aligned}
m_{110}-m_{160}= & -0.008+1.715(J-H) \\
& -0.554(J-H)^{2}+0.316(J-H)^{3},
\end{aligned}
$$

to convert the color change rate from $J-H$ to $m_{110}-m_{160}$, and we obtained $\Delta\left(m_{110}-m_{160}\right) / \Delta \tau \approx 0.02 \mathrm{mag} \mathrm{Gyr}^{-1}$. In the figure, we show the expected color difference in the giant branch with a \pm 2 Gyr age difference. It clearly shows that NGC 6287 and M92 have the same age to within 2 Gyr.

It should be noted that the fiducial sequences start to deviate from each other at a point $\approx 1 \mathrm{mag}$ above the base of RGB in the figure. It is not clear whether this is real, but it is more likely due to a statistical fluctuation with a small sample. ${ }^{8}$

\subsection{Reddening and Distance of NGC 6287}

We explore the reddening and the distance of NGC 6287 with respect to those of M92, whose values are relatively well known. In Table 5, we list the previous reddening and distance modulus estimates for both clusters. Published values range from $(m-M)_{0} \approx 14.60$ to $14.85 \mathrm{mag}$ for M92. Adopting $\left\langle V_{0}(\mathrm{RR})\right\rangle=15.04 \mathrm{mag}$ for M92 (Carney et al. 1992), the distance modulus of $14.60 \mathrm{mag}$ results in $\left\langle M_{V}(\mathrm{RR})\right\rangle=0.44 \mathrm{mag}$ and that of $14.85 \mathrm{mag}$ results in 0.19 mag. The $\left\langle M_{\mathrm{V}}(\mathrm{RR})\right\rangle=0.19 \mathrm{mag}$ for $[\mathrm{Fe} / \mathrm{H}]=-2.25$ is thought to be much too bright in comparison with the results of the Baade-Wesselink analysis of RR Lyrae variables (see, for example, Carney, Storm, \& Jones 1992) or the theoretical HB models (Lee, Demarque, \& Zinn 1990). For our purpose, therefore, we adopt the mean of Harris (1996) and Pont et al. (1998), and we obtain $(m-M)_{0}=14.60 \mathrm{mag}$ for M92. As may be seen in the table, the reddening and the distance modulus estimates for NGC 6287 do not agree in general.

We assume that the two clusters are identical and we compare the TO color and $m_{160+0.05}$. As shown in Tables 3

\footnotetext{
${ }^{8}$ A comparison with the ground-based observation of Davidge \& Courteau (1999) hints that our fiducial sequence for brightest red giant regime of NGC $6287\left(m_{160} \lesssim 12.5 \mathrm{mag}\right.$ in Table 3 or $m_{160}-m_{160+0.05} \lesssim$ $-6 \mathrm{mag}$ in Fig. 10) was possibly flawed by field star contamination.
}

and 4, $\left(m_{110}-m_{160}\right)_{\mathrm{TO}}=0.71 \mathrm{mag}$ and $m_{160+0.05}=18.20$ mag for NGC 6287, and $\left(m_{110}-m_{160}\right)_{\mathrm{TO}}=0.33 \mathrm{mag}$ and $m_{160+0.05}=18.10 \mathrm{mag}$ for M92.

Using the relations given in Table $6, E\left(m_{110}-m_{160}\right)=$ $0.59 E(B-V)$ and $A_{m_{160}}=0.59 E(B-V)$ for the mainsequence TO, we obtain $\Delta E\left(m_{110}-m_{160}\right)_{\mathrm{TO}}=0.38 \mathrm{mag}$ in the sense of NGC 6287 minus M92, and subsequently we obtain $E(B-V)=0.62 \mathrm{mag}$ for NGC 6287 after subtracting that of M92 $[E(B-V)=0.02 \mathrm{mag}]$. Our estimate of the interstellar reddening for NGC 6287 is in good agreement with that of Zinn (1980), Stetson \& West (1994), and Harris (1996). ${ }^{9}$

We compute the amount of interstellar absorption in the F160W passband by using these values, and we obtain the interstellar absorption-corrected magnitude $m_{160+0.05,0}=$ $17.83 \mathrm{mag}$ for NGC 6287 and $18.09 \mathrm{mag}$ for M92. The difference in $m_{160+0.05,0}$ magnitude between NGC 6287 and M92 is $-0.25 \mathrm{mag}$, indicating that NGC 6287 is located closer to the Sun than is M92. Using the mean $(m-M)_{0}$ for M92, we obtain $(m-M)_{0}=14.35 \mathrm{mag}$ for NGC 6287. Our estimate of the distance modulus for NGC 6287 agrees well with that of Stetson \& West (1994). If we adopt our distance modulus, the distance from the Sun becomes $7.4 \mathrm{kpc}$ and the Galactocentric distance $R_{\mathrm{GC}}$ becomes $1.6 \mathrm{kpc}$ if $R_{0}=8.0 \mathrm{kpc}$ (Reid 1993). NGC 6287 is clearly located in the Galaxy's central regions.

\section{DISCUSSION}

Our differential age determinations using the difference in color between the BRGB and the TO show that the most metal-poor inner halo cluster NGC 6287 appears to have essentially the same age as one of the oldest clusters in our Galaxy, M92. However, there are two potential problems that may affect our result. They are the possible differences in the elemental abundances between NGC 6287 and M92 and the existence of the temperature dependence on the interstellar extinction law in the HST NICMOS photometric system, especially in the F110W passband. These may affect the color difference between the BRGB and the TO, and subsequently they may mimic differences in relative ages.

\subsection{Effect of Elemental Abundances on the Relative Age Estimation}

Our results of the elemental abundance analysis of the two RGB stars in NGC 6287 by using high-resolution echelle spectra (Lee \& Carney 2001) indicate that NGC 6287 is slightly more metal-rich $(\delta[\mathrm{Fe} / \mathrm{H}] \approx 0.2 \mathrm{dex})$ than M92. In Figure 11, we show the effect of the different metallicity on the relative age estimation on the $(B-V, V$-plane using the model isochrones of Bergbusch \& VandenBerg (1992). As VandenBerg et al. (1990) have noted, their method using the difference in color between the BRGB and the TO is insensitive to metallicity and this is particularly true for the metal-poor regime. Although the model isochrones have slightly different oxygen abundances for each metallicity, the effect associated with the different metallicity is almost negligible in our result.

\footnotetext{
${ }^{9}$ Our interstellar reddening estimate for NGC 6287 is $\approx 0.2 \mathrm{mag}$ smaller than that of Schlegel, Finkbeiner, \& Davis (1998). Dutra \& Bica (2000) discussed that interstellar reddening estimates by Schlegel et al. for clusters near the Galactic plane appear to be larger than those based on stellar contents, possibly due to background dust.
} 


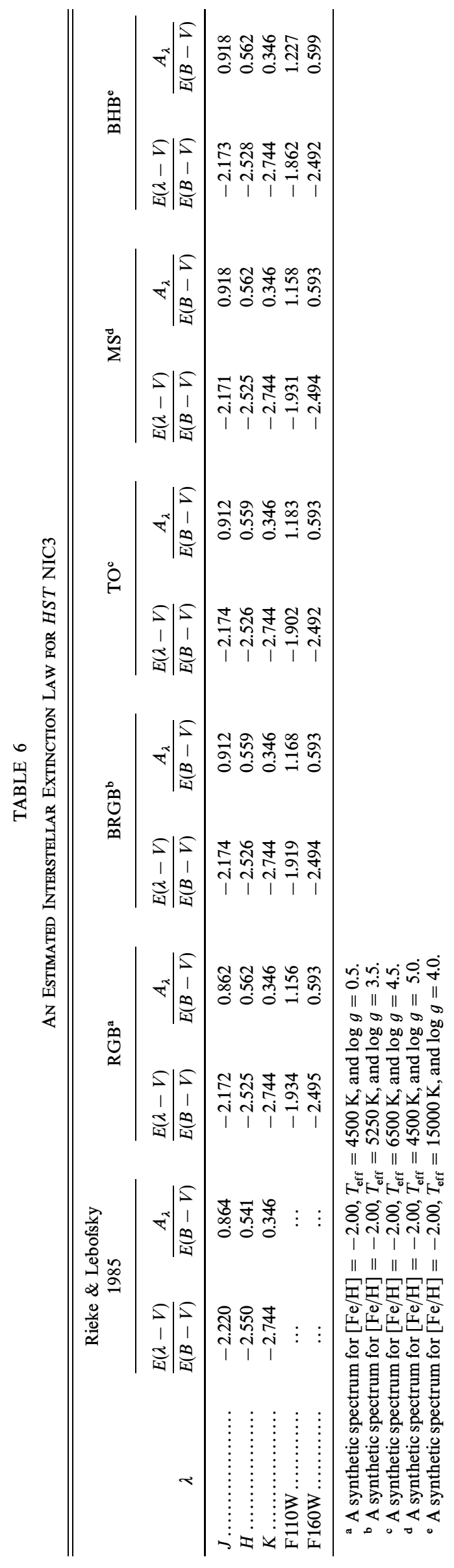




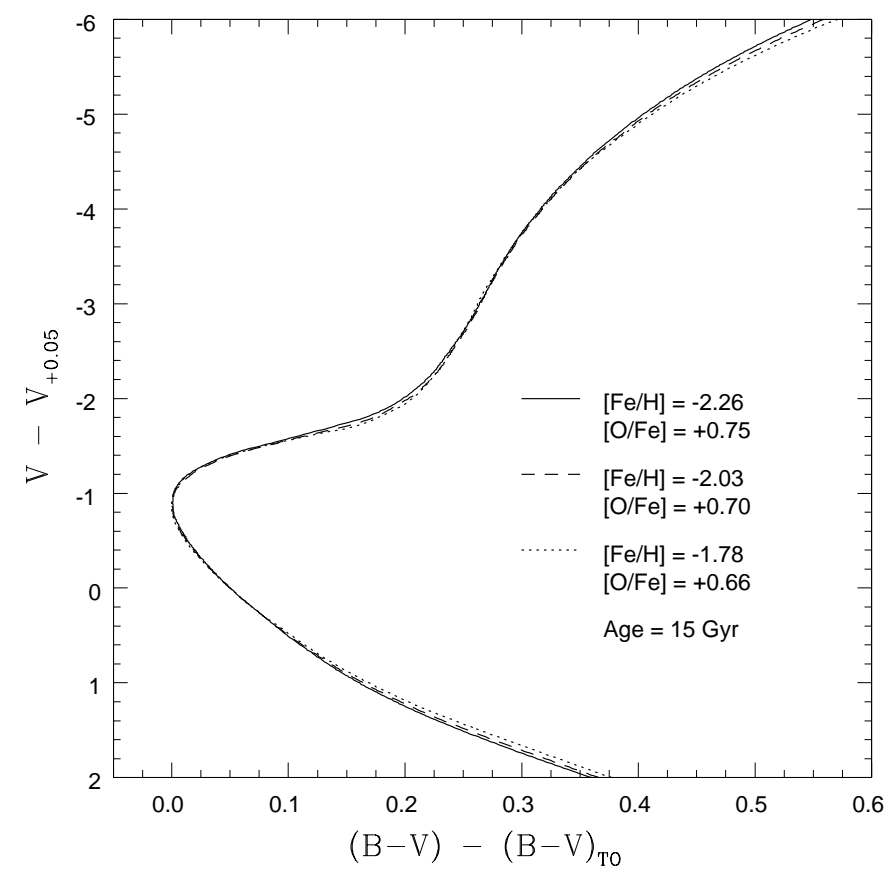

FIG. 11.-Theoretical model isochrones of Bergbusch \& VandenBerg (1992) for $[\mathrm{Fe} / \mathrm{H}]=-2.26,-2.03$, and -1.78 at an age of $15 \mathrm{Gyr}$. The difference in the metallicity is almost negligible in our relative age estimation for NGC 6287 and M92 using the color difference between the mainsequence turnoff and the base of the red giant branch.

The differences in the $\alpha$-element abundances, in particular the oxygen abundance, could also affect our results. Since oxygen has a high ionization potential, the difference in the oxygen abundance affects the color and the luminosity of TO stars and HB stars but not those of RGB stars, in the sense that higher oxygen abundance makes a redder and less luminous TO and a redder HB population. Oxygen also plays a vital role in the CNO cycle and hence in the TO and RGB luminosity levels (VandenBerg 1992). VandenBerg \& Stetson (1991) studied the effect of the oxygen abundance on the relative age estimations by using the method of VandenBerg et al. (1990). Their results suggested that changes in the oxygen abundance could mimic the age differences, in the sense that the higher oxygen abundance imitates the older age at a given metallicity. They also suggested that this effect is less important at the lowest metallicities and at the oldest ages.

The oxygen abundances of globular clusters are hard to determine, especially from a small sample. Oxygen abundances vary from star to star in a globular cluster, probably due to internal mixing during the RGB phase (see, for example, Langer, Hoffman, \& Sneden 1993; Kraft 1994). Thus in principle one must determine the oxygen abundance of the cluster based on unmixed stars, which should reflect the oxygen abundances of the presumably unmixed main-sequence and turnoff stars. The anticorrelation of sodium and oxygen abundances is a good means by which we may estimate if an individual cluster red giant has experienced significant mixing or not (Kraft 1994; Carney 1996). In particular, low sodium abundances $([\mathrm{Na} / \mathrm{Fe}] \leq 0)$ appear to be a good indicator of minimal mixing in globular cluster red giants. Our two stars have high $[\mathrm{Na} / \mathrm{Fe}]$ values, $\approx(+0.49)$, yet $[\mathrm{O} / \mathrm{Fe}]=+0.30 \pm 0.04$. The two stars may have undergone some mixing, but it is clear that their oxygen-to-iron ratios are enhanced, as is almost always the case for globular clusters. The four stars in M92 with [Na/ $\mathrm{Fe}] \leq 0.0$ have a mean $[\mathrm{O} / \mathrm{Fe}]$ value of $+0.22 \pm 0.10$ (Sneden et al. 1991; Shetrone 1996; Sneden, Pilachowski, \& Kraft 2000). One star (VII-18) is clearly deficient in oxygen, and the other three yield a mean value of $[\mathrm{O} / \mathrm{Fe}]$ of $+0.31 \pm 0.03$. It appears that the initial $[\mathrm{O} / \mathrm{Fe}]$ values of NGC 6287 and M92 may be very similar.

Enhancement of the other $\alpha$-elements increases the envelope opacity, and the model isochrones with enhanced $\alpha$-elements are shifted to fainter luminosities and cooler temperature. At low metallicities, in particular, the effects of $\alpha$-element enhancements could be treated by increasing overall heavy elements with a scaled-solar mixture (Chieffi, Straniero, \& Salaris 1991; VandenBerg et al. 2000). Carney (1996) claimed that the mean values of $[\mathrm{Si} / \mathrm{Fe}],[\mathrm{Ca} / \mathrm{Fe}]$, and $[\mathrm{Ti} / \mathrm{Fe}]$ of globular clusters are nearly constant $(\approx+0.30$ dex), regardless of their metallicities and ages. We therefore compare [Si/Fe], [Ca/Fe], and [Ti/Fe] between NGC 6287 and M92. We obtained $[\mathrm{Si} / \mathrm{Fe}]=+0.54 \pm 0.04, \quad[\mathrm{Ca} /$ $\mathrm{Fe}]=+0.18 \pm 0.06$, and $[\mathrm{Ti} / \mathrm{Fe}]=+0.11 \pm 0.05$ for the two RGB stars in NGC 6287, and the unweighted mean $[\alpha / \mathrm{Fe}]$ of NGC 6287 is $+0.28 \pm 0.03$ (Lee \& Carney 2001). Sneden et al. (2000) reported $[\mathrm{Si} / \mathrm{Fe}],[\mathrm{Ca} / \mathrm{Fe}]$, and $[\mathrm{Ti} / \mathrm{Fe}]$ for M92 RGB stars and they obtained $+0.59 \pm 0.04$, $+0.32 \pm 0.02$, and $+0.33 \pm 0.06$, respectively. The unweighted mean $[\alpha / \mathrm{Fe}]$ for M92 is $+0.41 \pm 0.02$, and it is +0.12 dex higher than that of NGC 6287. Using the scaling relation given by Chieffi et al. (1991), however, we obtain $Z=0.00030$ for NGC 6287 and 0.00021 for M92, and the influence on our relative age estimates due to the different chemical compositions is expected to be negligible.

\subsection{Effect of Reddening Dependence of the HST NICMOS Passbands on the Relative Age Estimation}

The precision of relative age estimates using the difference in color of the HST NICMOS photometric system could be slightly weakened by the differential color transformation effect coupled with the temperatures of the stars, particularly in the F110W passband. The problem arises because the HST filters are very broad (Calzetti et al. 1999). At a fixed value of the total interstellar absorption, a red star has more of its flux transmitted through the longwavelength (less extinguished) side of the filter than does a blue star, whose flux is stronger at shorter, more heavily extinguished, wavelengths. Our simulations with synthetic spectra at the various stellar evolutionary stages suggest that the interstellar extinction law for the F110W passband may depend on the temperature of the stars in the sense that the hotter stars suffer more interstellar extinction than the cooler stars in the F110W passband (see Appendix A) compared with standard $J H$ passbands. Subsequently the relative age estimate using the difference in the apparent color between the BRGB (i.e., the cooler temperature) and the main-sequence TO (i.e., the hotter temperature) of the heavily reddened clusters could be overestimated because the apparent color difference between the BRGB and the TO in such clusters could be smaller than the intrinsic color difference. This discrepancy increases with the reddening. In the case of NGC 6287, age relative to M92 could have been overestimated by $\approx 0.5 \mathrm{Gyr}$, or $\Delta\left(m_{110}-m_{160}\right)$ may have been underestimated by $\approx 0.01 \mathrm{mag}$, due to its high reddening. This differential reddening effect with tem- 
TABLE 7

Radial Velocities OF the Three RGB Stars in NGC 6287

\begin{tabular}{|c|c|c|c|c|c|}
\hline $\mathrm{ID}^{\mathrm{a}}$ & $V^{\mathrm{a}}$ & $B-V^{\mathrm{a}}$ & $\begin{array}{c}v_{r} \\
\left(\mathrm{~km} \mathrm{~s}^{-1}\right)\end{array}$ & $\begin{array}{c}\sigma \\
\left(\mathrm{km} \mathrm{s}^{-1}\right)\end{array}$ & $\mathrm{S} / \mathrm{N}$ \\
\hline $1491 \ldots \ldots$ & 14.22 & 1.68 & -290.04 & 0.94 & 95 \\
\hline $1387 \ldots \ldots$ & 14.28 & 1.88 & -294.06 & 0.89 & 95 \\
\hline $1191 \ldots \ldots$ & 14.58 & 1.64 & -282.31 & 0.67 & 40 \\
\hline
\end{tabular}

${ }^{\text {a }}$ Stetson \& West 1994.

perature of the source could lead to a larger error in relative age estimates, if this effect is not taken into account, than the difference in the chemical composition between NGC 6287 and M92, as we discussed above.

The existence of the differential color transformation effect with the temperature of the source has not been studied observationally; however, our indirect demonstration strongly suggests its existence (see Appendix A). If this differential color transformation with the temperature of the source exists, it could be a serious problem in interpreting the HST NICMOS data since HST NICMOS has been used for observations of heavily reddened objects. More extensive study associated with the differential color transformation effect of HST NICMOS is encouraged.

\subsection{Did NGC 6287 Form in the Inner Halo?}

Stetson \& West (1994) noted that NGC 6287 may have sufficient kinetic energy to reach at least near the solar circle, and they questioned whether it is a genuine inner halo cluster. Therefore, it is worthwhile to consider the apogalacticon distance of NGC 6287 since it should be related to the cluster's origin.

With the new radial velocity measurement by Lee \& Carney (2001; see also Table 7), we have integrated the motion of NGC 6287 by using the method described by Dinescu, Girard, \& van Altena (1999). For our calculations, we adopt a Galactic potential model given by Paczyński (1990), but no tangential motions are included since the proper motion of the cluster is not yet known. Our calculations indicate that its apogalacticon distance is $R_{a} \approx 5.5$ $\mathrm{kpc},{ }^{10}$ and this is smaller than the previous estimate by Stetson \& West (1994) despite our larger radial velocity measurement. It should be noted, however, that our estimate of its apogalacticon distance is the minimum apogalacticon distance since no part of the cluster's tangential velocity is considered. Our calculations suggest that since NGC 6287 is currently located deep inside the Galactic potential well, at least an additional tangential velocity of $190 \mathrm{~km} \mathrm{~s}^{-1}$ is required to make it reach as far as the solar circle. A tangential velocity this large will result in a larger proper motion $\left(\approx 5.4\right.$ mas $\left.\mathrm{yr}^{-1}\right)$, and this large proper motion could be easily determined using $H S T$ with a several years' time span. Considering the importance of proper motion in deriving appropriate orbital parameters, a proper-motion measurement for NGC 6287 is strongly encouraged (see Table 7).

Our minimum apogalacticon distance of NGC 6287

${ }^{10}$ D. Dinescu (2000, private communication) obtained a similar apogalacticon distance scale for NGC 6287 by using our radial velocity measurement and Paczyński's Galactic potential model. In the case of using the radial velocity of Hesser et al. (1986), the apogalacticon distance of NGC 6287 is $\approx 4 \mathrm{kpc}$. appears to be too large to classify it as a genuine inner halo with $R_{\mathrm{GC}} \leq 3 \mathrm{kpc}$. It should be noted that, however, the Galactic spheroidal half-light radius is $\approx 3 \mathrm{kpc}$ (Gilmore, Wyse, \& Kuijken 1989) and the apogalacticon distance of $5.5 \mathrm{kpc}$ must be inside the Galactic spheroid. The small half-mass radius of NGC $6287\left(r_{h}=1.3 \mathrm{pc}\right)$ also suggests that it must be related to the inner part of our Galaxy at the time of formation. Van den Bergh (1994) suggested that the half-mass radius ${ }^{11}$ of the globular cluster system is correlated with the Galactocentric distance, in the sense that the half-mass radius increases with the Galactocentric distance (see his Fig. 1), and NGC 6287 appears to agree with this correlation. He suggested that this correlation is mainly related to a physical correlation that was established at the time of cluster formation (see also McLaughlin 2000). Therefore, NGC 6287 is most likely an inner halo cluster as van den Bergh (1993) suggested, unless its tangential velocity is extremely large.

\section{CONCLUSION}

We have presented HST NIC3 photometry of the inner halo globular cluster NGC 6287 and the intermediate halo globular cluster M92. For HST NIC3, the undersampling combined with the variation in the intrapixel sensitivity across the individual pixels is the dominant source of the photometric errors. We have discussed that a dithering scheme with a subpixel offset could significantly reduce the discrepancies. We have shown that standard system magnitude calibrations for the HST NIC3 photometric system are somewhat uncertain because of the undersampled PSFs with the variation in the intrapixel sensitivity across the individual pixels, but the relative magnitude comparison between globular clusters can be achieved with good accuracy. This is especially true in the case of the mean loci of the clusters color-magnitude diagrams, which are more important for our process than the measurements of the individual stars.

Our measurements range from the $\mathrm{RGB}$ to $\approx 3 \mathrm{mag}$ below the TO, allowing us to define accurate mean loci for the two clusters. Our relative age estimate for NGC 6287 and M92 using the color difference between the mainsequence TO and the BRGB (VandenBerg et al. 1990) showed that they essentially have the same ages to within \pm 2 Gyr. The slightly different chemical compositions between the two clusters do not significantly affect our result. Our simulations using synthetic spectra have shown that the temperature dependence on the interstellar reddening law for the HST NICMOS photometric system could be a more serious problem in the relative age estimation using the color difference between the TO and the BRGB. However, this effect adds less than $1 \mathrm{Gyr}$ uncertainty to our relative age estimation. The effect will be more serious for more heavily reddened clusters.

We have estimated the relative interstellar reddening toward NGC 6287 (field F1) and the relative distance modulus with respect to those of M92. We obtained $E(B-V)=0.62$ and $(m-M)_{0}=14.35$ for NGC 6287, assuming $(m-M)_{0}=14.60$ for M92. Our photometry suggested that there exists a differential reddening across

\footnotetext{
${ }^{11}$ See, for example, Spitzer \& Thuan (1972) for pseudoinvariance of the half-mass radius.
} 
NGC 6287 with an amount of $\Delta E(B-V)=0.07-0.09$ mag, and this is consistent with the previous study of Stetson \& West (1994). The Galactocentric distance of NGC 6287 becomes $R_{\mathrm{GC}}=1.6 \mathrm{kpc}$ if $R_{0}=8.0 \mathrm{kpc}$. Using the new radial velocity measurement (Lee \& Carney 2001), our estimate of the minimum apogalacticon distance of NGC 6287 is $R_{a} \approx 5.5 \mathrm{kpc}$, indicating that NGC 6287 is probably an inner halo cluster.

Our results agree with the idea that the globular cluster formation must have been triggered everywhere at the same time in our Galaxy. Since Harris et al. (1997) claimed that the very remote halo cluster NGC $2419\left(R_{\mathrm{GC}}=90 \mathrm{kpc}\right)$ and M92 essentially have the same age, our result extends this to NGC 6287. A more comprehensive study with an expanded sample of metal-poor inner halo clusters, such as NGC 6293,6333 , and 6541, will reveal the more detailed chronology of the inner halo. Our preliminary results of $H S T$
PC2 photometry of NGC 6293 and NGC 6541 also suggest that these two clusters have the same ages as M92. We will subsequently discuss HST PC2 photometry of NGC 6293 and NGC 6541.

This is part of Ph.D. thesis work of J.-W. Lee at the University of North Carolina at Chapel Hill. J.-W. Lee wishes to thank T. Davidge, D. Dinescu, and R. Rood for their kind discussion. This research was supported by the National Aeronautics and Space Administration (NASA) grant number GO-07318.04-96A from the Space Telescope Science Institute, which is operated by the Association of Universities for Research in Astronomy, Inc., under NASA contract NAS 5-26555. We also thank the National Science Foundation for financial support via grants AST 96-19381 and AST 98-0531 to the University of North Carolina.

\section{APPENDIX A}

\section{ESTIMATION OF EXTINCTION LAW FOR HST NICMOS F110W AND F160W PASSBANDS}

We estimate the theoretical interstellar extinction law for HST NICMOS F110W and F160W passbands by using the method described by Buser \& Kurucz (1978) and the synthetic spectra of Kurucz (1993). The individual magnitudes are defined by

$$
m_{i}=-2.5 \log \int_{0}^{\infty} F(\lambda) S_{i}(\lambda) d \lambda,
$$

where $F(\lambda)$ is the stellar flux per unit wavelength and $S_{i}(\lambda)$ is the response function for the passband $i$. For our calculations, we adopt $S_{i}(\lambda)$ from Bessell \& Brett (1988), Bessell (1990), and Calzetti et al. (1999). The zero points are calibrated using the synthetic spectrum for Vega, which has zero magnitude in all passbands. Then the individual magnitudes with the interstellar extinction, $m_{i}^{\mathrm{A}}$, for each passband, $i$, are defined by

$$
m_{i}^{A}=-2.5 \log \int_{0}^{\infty} F(\lambda) S_{i}(\lambda) 10^{-A(\lambda) / 2.5} d \lambda
$$

where $A(\lambda)$ is the interstellar extinction function and we adopted that of Mathis (1990) for $R_{V}=3.1$. (It should be noted that real individual magnitudes should involve response functions of the detector and optics.)

We compute individual magnitudes at the five main evolutionary phases, RGB ( $T_{\text {eff }}=4500 \mathrm{~K}$ and $\left.\log g=0.5\right)$, BRGB $\left(T_{\text {eff }}=5250 \mathrm{~K}\right.$ and $\left.\log g=3.5\right)$, TO $\left(T_{\text {eff }}=6500 \mathrm{~K}\right.$ and $\left.\log g=4.5\right)$, MS $\left(T_{\text {eff }}=4500 \mathrm{~K}\right.$ and $\left.\log g=5.0\right)$, and BHB $\left(T_{\text {eff }}=\right.$ $15000 \mathrm{~K}$ and $\log g=4.0$ ), using synthetic spectra for $[\mathrm{Fe} / \mathrm{H}]=-2.0$ (Kurucz 1993). In Figure 12, we show these synthetic spectra and the response functions for HST NICMOS F110W and F160W passbands and the standard $J H$ passbands (see also Appendix B). We also show the interstellar extinction function of Mathis (1990) normalized at $V$ passband $(\lambda=0.55 \mu \mathrm{m})$. It should be noted that the slopes of the synthetic spectra do not change significantly with surface gravities. However, the slopes of the synthetic spectra change significantly with temperature in the F110W passband, particularly in the wavelength range $\lesssim 1.1 \mu \mathrm{m}$, and they depend on the temperature of the source. Also, the interstellar extinction function changes its slope at the shorter wavelength region, suggesting that the interstellar extinction law for the HST NICMOS F110W passband depends on the temperature of the source.

In Table 6, we present the results of the synthetic interstellar extinction law for HST NICMOS F110W and F160W passbands and for the standard $J H K$ passbands. We assume $E(K-V) / E(B-V)=-2.744$ for our calculations using a method described by Rieke \& Lebofsky (1985). As comparison values for our theoretical calculations in JHK passbands, we also list the observational values of Rieke \& Lebofsky (1985). As shown in the table, our theoretical values agree well with the observational values of Rieke \& Lebofsky (1985). The theoretical calculations indicate that the absorption coefficient $A_{\lambda}$ is almost independent of the temperature or the surface gravity in $J H K$ passbands and in the HST NICMOS F160W passband. However, the interstellar absorption coefficient in the F110W passband, $A_{\mathrm{F} 110 \mathrm{~W}}$, appears to depend on the temperature in the sense that $A_{\mathrm{F} 110 \mathrm{w}}$ increases with temperature. Our calculations show that the difference in $A_{\mathrm{F} 110 \mathrm{w}} / E(B-V)$ between RGB and TO stars could be as large as $0.02 \mathrm{mag}$, suggesting the TO stars (i.e., hotter stars) suffer more interstellar extinction than do the RGB stars (i.e., cooler stars). If this differential extinction with the temperature of the source is correct, then the discrepancy in the $\left(m_{\mathrm{F} 110 \mathrm{w}}-m_{\mathrm{F} 160}\right)$ colors between the TO and the RGB could be as large as $0.02 \mathrm{mag}$ for the interstellar reddening $E(B-V)=1.0$, in the sense that the difference in the observed color between the TO and the RGB is $0.02 \mathrm{mag}$ smaller than that in intrinsic (dereddened) color. 


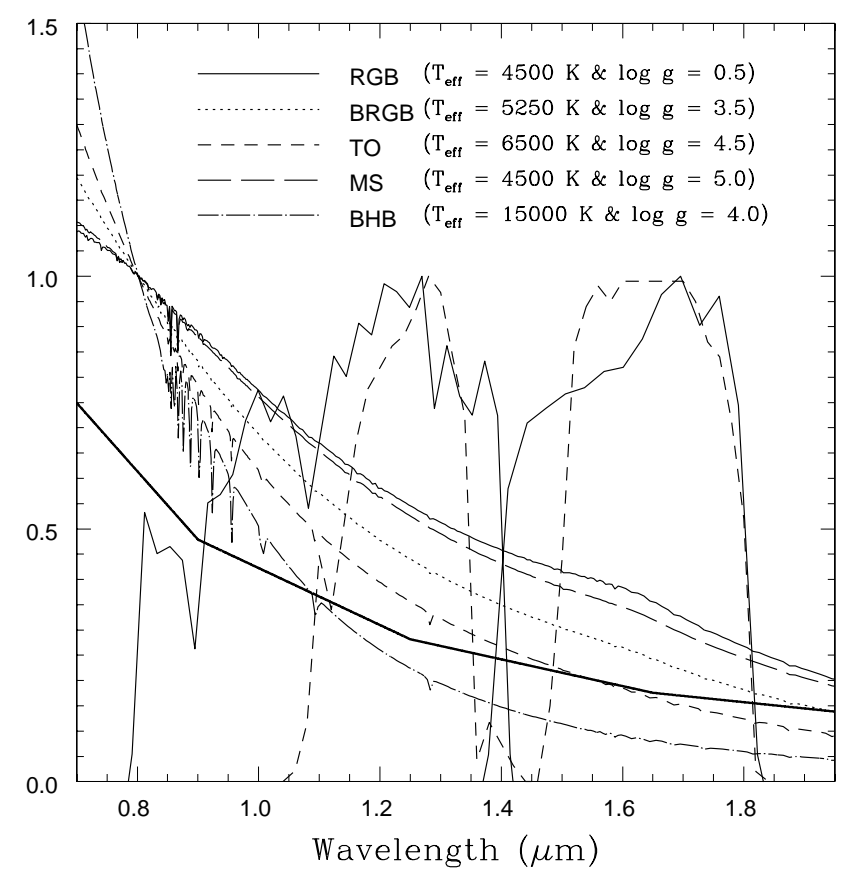

Fig. 12. - Comparison of filter passbands between HST NIC3 (Calzetti et al. 1999) and the JH system (Bessell \& Brett 1988). The HST NIC3 filter system is represented by solid lines, and the $J H$ filter system, by dashed lines. A thick solid line represents the interstellar extinction law by Mathis (1990) normalized at the $V$ passband $(\lambda=0.55 \mu \mathrm{m})$. The synthetic spectra (Kurucz 1993) at various stellar evolutionary stages for the old metal-poor globular cluster are also indicated. In the figure, the synthetic spectra are normalized at $\lambda=0.80 \mu \mathrm{m}$.

\section{APPENDIX B}

\section{THE INTERSTELLAR REDDENING DEPENDENCE ON TRANSFORMATIONS BETWEEN THE HST NICMOS F110W/F160W PHOTOMETRIC SYSTEM AND THE GROUND-BASED JH SYSTEM}

The photometric transformation relations between the HST WFPC2 photometric system and the ground-based BVI photometric system (see, for example, Holtzman et al. 1995), allow us to relate HST observations to the ground-based observations. In fact, these photometric systems are used often without any distinction. However, to derive the transformation relations between two different photometric systems is not a trivial matter, particularly when the central wavelength and the bandwidth of the photometric systems differ significantly.

The transformation relations between the HST NICMOS photometric system and the standard $J H$ photometric system are frequently required since the most of the theoretical framework has been established in the standard $J H$ passbands. It is known, however, that the HST NICMOS F110W passband is more than twice as broad as the $J$ passband and extends 0.3 $\mu$ m bluer (see Fig. 12). The HST NICMOS F160W passband is about $35 \%$ broader than the $H$ passband and extends $0.1 \mu \mathrm{m}$ bluer. Because of these very different characteristics, the transformation relations between the HST NICMOS photometric system and the ground-based $J H$ photometric systems might not be independent of the temperature of the sources. Stephens et al. (2000) and Origlia \& Leitherer (2000) derived transformation relations, and they suggested that their relations may depend on the metallicity. But even this is not the whole story. The transformation relations may depend not only on the metallicity but also on the interstellar reddening. Because the HST NICMOS photometric system and the ground-based photometric system have different central wavelengths and bandwidths, the governing interstellar extinction laws for each filter system are different, as we demonstrated in Appendix A. This is especially true for F110W and $J$ passbands.

We demonstrate this effect by comparing our measurements with those of Davidge \& Courteau (1999). In NGC 6287, a small area in our field F1 overlaps with the fields of Davidge \& Courteau (1999) but, unfortunately, there is no common area for M92. We show the overlapped area in Figure $1 a$ with a solid line. We select 77 common stars with our estimated measurement errors $\sigma\left(m_{110}-m_{160}\right) \leq 0.10$ mag and derive the transformation relations between the two photometric systems,

$$
\begin{aligned}
(J-H)_{\mathrm{DC} 99} & =(0.838 \pm 0.010)\left(m_{110}-m_{160}\right)-(0.060 \pm 0.008), \\
H_{\mathrm{DC} 99} & =m_{160}+(0.064 \pm 0.014)\left(m_{110}-m_{160}\right)+(0.015 \pm 0.010),
\end{aligned}
$$

where $(J-H)_{\mathrm{DC} 99}$ and $H_{\mathrm{DC} 99}$ refer to $J-H$ colors and $H$ magnitudes of Davidge \& Courteau (1999). (The errors are those of the mean.) In Figure 13, we compare our CMD using the transformed color and magnitude with that of Davidge \& Courteau (1999).

Using these relations, we obtain the transformed colors and magnitudes for M92 stars, and we compare them with the ground-based observation of Davidge \& Courteau (1999) in Figure 14. The figure indicates that the transformation relations 


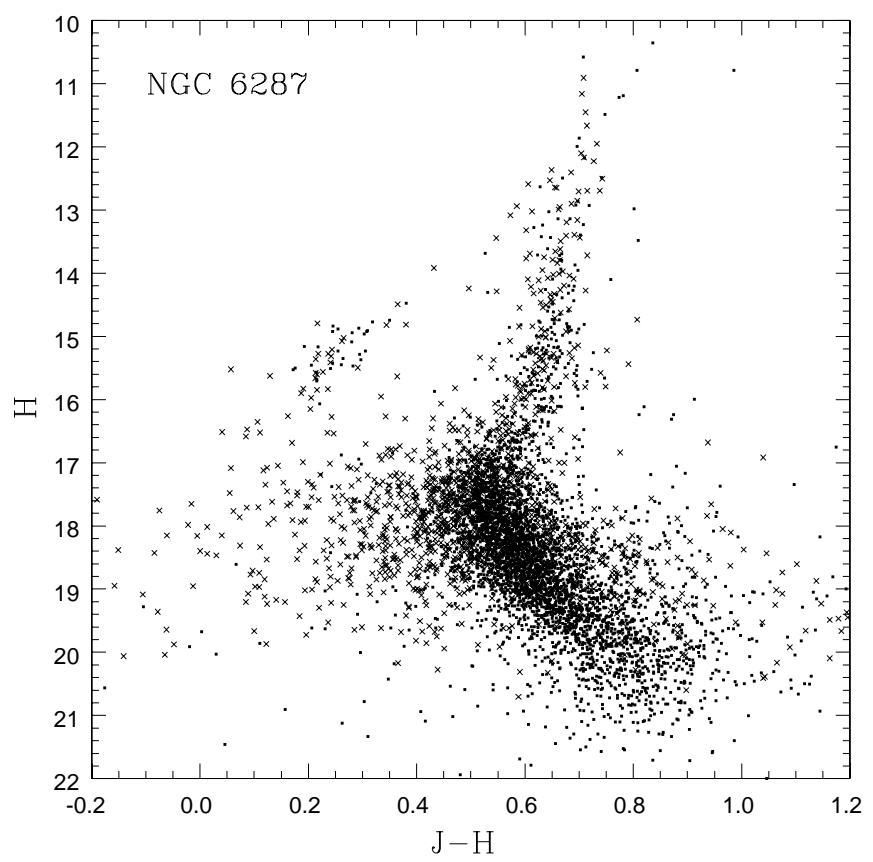

FIG. 13. - Comparison of the color-magnitude diagrams of NGC 6287 of the current study [after converting $\left(m_{110}-m_{160}, m_{160}\right)$ into $\left.(J-H, H)\right]$ by using the transformation relations (eqs. [B1] in Appendix B and in Davidge \& Courteau 1999). The current study is represented by dots, and that of Davidge \& Courteau by plus signs.

derived from NGC 6287 fail to transform our HST NIC3 photometry to the ground-based photometry. Assuming the clusters' CMDs should be identical, we argue that the mismatch is caused by the reddening effects on the transformation of NIC3 photometry to the $J H$ system. Also, the influence of the interstellar reddening in $m_{110}-m_{160}$ is more important than in $J-H$ because in the $H S T$ photometric system the bandwidths are broader and the cutoff wavelengths in F110W and F160W are shorter than those of the standard JH photometric system. This effect may result in some serious problems when HST NICMOS observations have been performed for heavily reddened objects. As a result, the slope and the zero points of the transformation relations depend on the metallicity, the temperature, and the interstellar reddening.

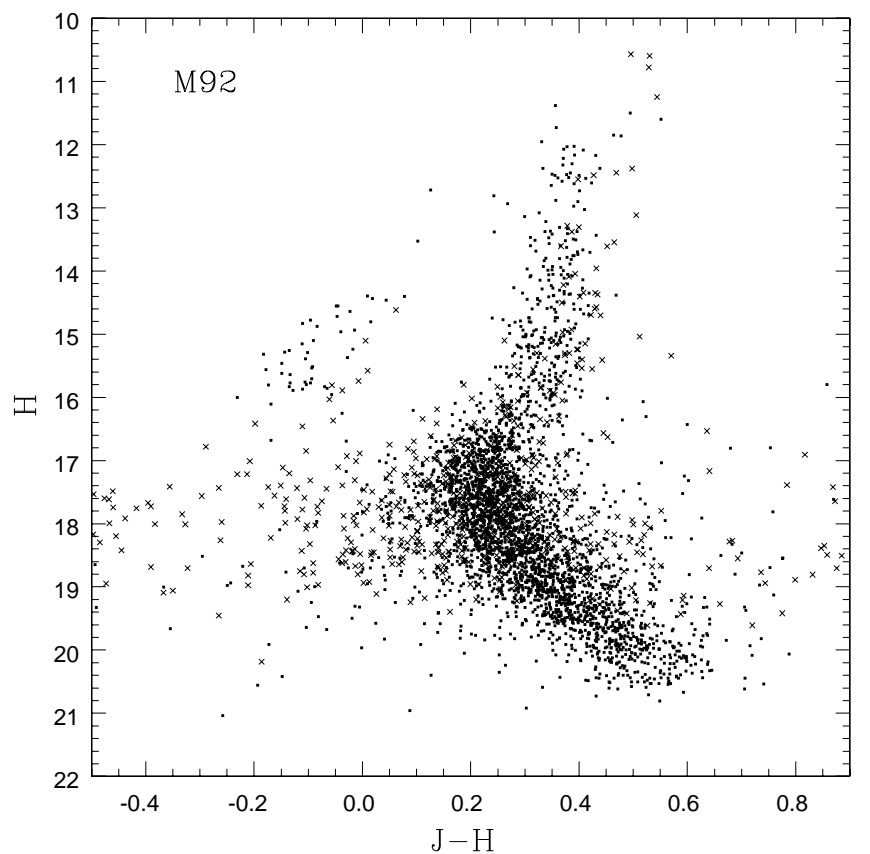

FIG. 14.-Comparison of the color-magnitude diagrams of M92 of the current study and Davidge \& Courteau (1999) using the transformation relations (eqs. [B1]) found in NGC 6287. The conversion relations found in the reddened cluster NGC $6287[E(B-V)=0.62]$ fail to agree in the slightly reddened cluster M92 $[(E(B-V)=0.02]$ because of the interstellar reddening dependence on transformations between the HST NICMOS photometric system and the ground-based $J H$ photometric system. The current study is represented by dots, and that of Davidge \& Courteau, by plus signs. 
Bell, R. A. 1992, MNRAS, 257, 423

Bergbusch, P. A., \& VandenBerg, D. A. 1992, ApJS, 81, 163

Bessell, M. S. 1990, PASP, 102, 1181

Bessell, M. S., \& Brett, J. M. 1988, PASP, 100, 1134

Bessell, M. S., Castelli, F., \& Plez, B. 1998, A\&A, 333, 231

Buonanno, R., Corsi, C. E., Zinn, R., Fusi Pecci, F., Hardy, E., \& Suntzeff, N. B. 1998, ApJ, 501, L33

Burstein, D., \& Heiles, C. 1982, AJ, 87, 1165

Buser, R., \& Kurucz, R. L. 1978, A\&A, 70, 555

Calzetti, D. et al. 1999, NICMOS Instrument Handbook, Version 3.0, ed. D. Calzetti, et al. (Baltimore: STScI)

Carney, B. W. 1996, PASP, 108, 900

Carney, B. W., Storm, J., \& Jones, R. V. 1992, ApJ, 386, 663

Carney, B. W., Storm, J., Trammell, S. R., \& Jones, R. V. 1992, PASP, 104, 44

Chaboyer, B., Demarque, P., \& Sarajedini, A. 1996, ApJ, 459, 558

Chieffi, A., Straniero, O., \& Salaris, M. 1991, in ASP Conf. Ser. 13, The Formation and Evolution of Star Clusters, ed. K. Janes (San Francisco: ASP), 219

Colina, L., \& Rieke, M. J. 1997, in HST Calibration Workshop, ed. S. Casertano, R. Jedrzejewski, T. Keyes, \& M. Stevens (Baltimore: STScI), 182

Davidge, T. L. 1998, AJ, 116, 1744

Davidge, T. L., \& Courteau, S. 1999, AJ, 117, 1297

Dinescu, D. I., Girard, T. M., \& van Altena, W. F. 1999, AJ, 117, 1792

Djorgovski, S. 1993, in ASP Conf. Ser. 50, Structure and Dynamics of Globular Clusters, ed. S. G. Djorgovski \& G. Meylan (San Francisco: ASP), 373

Dutra, C. M., \& Bica, E. 2000, A\&A, 359, 347

Eggen, O. J., Lynden-Bell, D., \& Sandage, A. 1962, ApJ, 136, 748 (ELS)

Fullton, L. K., Stetson, P. B., \& Carney, B. W. 1999, Ap\&SS, 265, 361

Gilmore, G., Wyse, R. F. G., \& Kuijken, K. 1989, ARA\&A, 27, 555

Gratton, R. G., Fusi Pecci, F., Carretta, E., Clementini, G., Corsi, C. E., \&

Lattanzi, M. 1997, ApJ, 491, 749

Grillmair, C. J., et al. 1998, AJ, 115, 144

Hamann, F., \& Ferland, G. 1999, ARA\&A, 37, 487

Harris, W. E. 1996, AJ, 112, 1487

Harris, W. E., et al. 1997, AJ, 114, 1030

Held, E. V., Saviane, I., Momany, Y., \& Carraro, G. 2000, ApJ, 530, L85

Hesser, J. E., Shawl, S. J., \& Meyer, J. E. 1986, PASP, 98, 403

Holtzman, J. A., Burrows, J., Casertano, S. Hester, J., Trauger, J. T., Waston, A. M., \& Worthey, G. 1995, PASP, 107, 1065

Johnson, J. A., Bolte, M., Stetson, P. B., Hesser, J. E., \& Somerville, R. S. 1999, ApJ, 527, 199

Kraft, R. P. 1994, PASP, 106, 553

Krist, J. E., Golimowski, D. A., Schroeder, D. J., \& Henry, T. J. 1998, PASP, 110, 1046

Krist, J. E., \& Hook, R. 2000, The Tiny Tim User's Guide, Version 5.0a (Baltimore: STScI)

Kurucz, R. L. 1993, CD-ROM 13, ATLAS9 Stellar Atmosphere Programs and $2 \mathrm{~km} / \mathrm{s}$ Grid (Cambridge: Smithsonian Astrophys. Obs.)

Langer, R. E., Hoffman, R., \& Sneden, C. 1993, PASP, 105, 301

Lauer, T. R. 1999, PASP, 111, 1434

\section{EFERENCES}

Layden, A. C., \& Sarajedini, A. 2000, AJ, 119, 1760

Lee, J.-W., \& Carney, B. W. 1999, AJ, 117, 2868 2001, in preparation

Lee, Y.-W., Demarque, P., \& Zinn, R. 1990, ApJ, 350, 155 1994, ApJ, 423, 248

Mathis, J. S. 1990, ARA\&A, 28, 37

McLaughlin, D. E. 2000, ApJ, 539, 618

Mighell, K. J. 1997, AJ, 114, 1458

Mighell, K. J., \& Burke, C. J. 1999, AJ, 118, 366

Mighell, K. J., \& Rich, R. M. 1996, AJ, 111, 777

Montegriffo, P., Bellazzini, M., Ferraro, F. R., Martins, D., Sarajedini, A., $\&$ Fusi Pecci, F. 1998, MNRAS, 294, 315

Origlia, L., \& Leitherer, C. 2000, AJ, 119, 2018

Paczyński, B. 1990, ApJ, 348, 485

Pont, F., Mayor, M., Turon, C., \& Vandenberg, D. A. 1998, A\&A, 329, 87

Reed, B. C., Hesser, J. E., \& Shawl, S. J. 1988, PASP, 100, 545

Reid, I. N. 1997, AJ, 114, 161

Reid, M. J. 1993, ARA\&A, 31, 345

Richer, H. B., et al. 1996, ApJ, 463, 602

Rieke, G. H., \& Lebofsky, M. J. 1985, ApJ, 288, 618

Rosenberg, A., Saviane, I., Piotto, G., \& Aparicio, A. 1999, AJ, 118, 2306

Sarajedini, A., Chaboyer, B., \& Demarque, P. 1997, PASP, 109, 1321

Schlegel, D. J., Finkbeiner, D. P., \& Davis, M. 1998, ApJ, 500, 525

Searle, L., \& Zinn, R. 1978, ApJ, 225, 357 (SZ)

Shetrone, M. D. 1996, AJ, 112, 1517

Sneden, C., Kraft, R. P., Prosser, C. F., \& Langer, G. E. 1991, AJ, 102, 2001

Sneden, C., Pilachowski, C. A., \& Kraft, R. P. 2000, AJ, 120, 135

Spitzer, L., \& Thuan, T. X. 1972, ApJ, 175, 31

Stephens, A. W., Frogel, J. A., Ortolani, S., Davis, R., Jablonka, P., Renzini, A., \& Rich, R. M. 2000, AJ, 119, 419

Stetson, P. B. 1994, PASP, 106, 250

. 1995, DAOPHOTII User's Manual (Victoria: Dominion Astrophys. Obs.)

Stetson, P. B., VandenBerg, D. A., \& Bolte, M. 1996, PASP, 108, 560

Stetson, P. B., \& West, M. J. 1994, PASP, 106, 726

Storrs, A., Hook, R., Stiavelli, M., Hanley, C., \& Freudling, W. 1999, Instrument Science Report NICMOS-99-005 (Baltimore: STScI)

Trager, S. C., King, I. R., \& Djorgovski, S. 1995, AJ, 109, 218

Turner, A. M. 1995, Cooking with ALLFRAME (Victoria: Dominion Astrophys. Obs.)

VandenBerg, D. A. 1992, ApJ, 391, 685

VandenBerg, D. A., Bolte, M., \& Stetson, P. B. 1990, AJ, 100, 445

VandenBerg, D. A., \& Stetson, P. B. 1991, AJ, 102, 1043

VandenBerg, D. A., Swenson, F. J., Rogers, F. J., Iglesias, C. A., \& Alexander, D. R. 2000, ApJ, 532, 430

van den Bergh, S. 1993, ApJ, 411, 178 1994, AJ, 108, 2145 1996, PASP, 108, 986

Webbink, R. F. 1985, in IAU Symp. 113, Dynamics of Star Clusters, ed. J. Goodman \& P. Hut (Dordrecht: Reidel), 541

Yan, L., McCarthy, P. J., Storrie-Lombardi, L. J., \& Weyman, R. J. 1998, ApJ, 503, L19

Zinn, R. 1980, ApJS, 42, 19 